MATHEMATICS OF COMPUTATION

Volume 72, Number 243, Pages 1281-1303

S 0025-5718(02)01468-0

Article electronically published on October 18, 2002

\title{
ANALYSIS OF MULTILEVEL METHODS FOR EDDY CURRENT PROBLEMS
}

\author{
R. HIPTMAIR
}

\begin{abstract}
In papers by Arnold, Falk, and Winther, and by Hiptmair, novel multigrid methods for discrete $\boldsymbol{H}(\mathbf{c u r l} ; \Omega)$-elliptic boundary value problems have been proposed. Such problems frequently occur in computational electromagnetism, particularly in the context of eddy current simulation.

This paper focuses on the analysis of those nodal multilevel decompositions of the spaces of edge finite elements that form the foundation of the multigrid methods. It provides a significant extension of the existing theory to the case of locally vanishing coefficients and nonconvex domains. In particular, asymptotically uniform convergence of the multigrid method with respect to the number of refinement levels can be established under assumptions that are satisfied in realistic settings for eddy current problems.

The principal idea is to use approximate Helmholtz-decompositions of the function space $\boldsymbol{H}(\operatorname{curl} ; \Omega)$ into an $H^{1}(\Omega)$-regular subspace and gradients. The main results of standard multilevel theory for $H^{1}(\Omega)$-elliptic problems can then be applied to both subspaces. This yields preliminary decompositions still outside the edge element spaces. Judicious alterations can cure this.
\end{abstract}

\section{INTRODUCTION}

The eddy current model (see 22) leads to degenerate parabolic initial-boundary value problems. Though the equations are initially posed on the entire space $\mathbb{R}^{3}$, we can switch to a bounded domain by introducing an artificial boundary sufficiently removed from the region of interest. This is common in engineering simulations 24] and results in a bounded and polyhedral computational domain $\Omega \subset \mathbb{R}^{3}$. For the sake of stability, time-stepping schemes have to be $L$-stable and stiffly accurate, requirements that can only be met by implicit schemes. When we restrict our attention to linear, isotropic media, each time step entails the solution of a discretized version of the degenerate elliptic boundary value problem

$$
\operatorname{curl} \chi \operatorname{curl} \mathbf{u}+\beta \mathbf{u}=\mathbf{f} \quad \text { in } \Omega, \quad \mathbf{u} \times \mathbf{n}=0 \quad \text { on } \partial \Omega .
$$

The coefficients $\chi$ (magnetic susceptibility) and $\beta$ belong to $L^{\infty}(\Omega)$ and are nonnegative almost everywhere. For physical reasons the right hand side $\mathbf{f} \in \boldsymbol{L}^{2}(\Omega)$ has to be divergence free. Besides, $\chi$ is uniformly positive, i.e., for some $\underline{\chi}$, $\bar{\chi}>0$ holds $0<\underline{\chi} \leq \chi \leq \bar{\chi}$ a.e. in $\Omega$.

Received by the editor November 6, 2000 and, in revised form, August 13, 2001 and September 19, 2001.

2000 Mathematics Subject Classification. Primary 65N55, 65N30, 35Q60.

Key words and phrases. Edge elements, multilevel methods, stable BPX-type splittings, multigrid in $\boldsymbol{H}(\mathbf{c u r l} ; \Omega)$, Helmholtz-decomposition.

This work was supported by DFG as part of SFB 382 .

(C)2002 American Mathematical Society 
The label "degenerate" is due to the behavior of the coefficient $\beta \in L^{\infty}(\Omega)$, which, in physical terms, is a scalar multiple of the conductivity $\sigma$ divided by the length of the time step. Usually, there is a crisp distinction between conducting regions $\Omega_{C}$, where $\sigma$ is bounded away from zero, and insulating regions, where $\sigma=0$. Thus, we will take for granted that $\beta \geq \underline{\beta}>0$, for some bound $\underline{\beta}>0$, wherever $\beta \neq 0$. Often, the material parameters vary only moderately and smoothly inside and outside the conductor. Hence, for the sake of lucidity and without departing too much from realistic situations, we can make the assumption that $\chi=\chi_{0}>0$ everywhere and $\beta=\beta_{0}>0$ in $\Omega_{C}$.

It is important to note that the really interesting quantity is curl u. This is why we can use an ungauged formulation as in (1.1), which does not impose a constraint on div $\mathbf{u}$ outside $\Omega_{C}$. Obviously, this forfeits uniqueness of the solution in parts of the domain where $\beta=0$, but the solution for curl $\mathbf{u}$ remains unique everywhere. Inside the conductor, where $\beta>0$, we get a unique $\mathbf{u}$.

Problem (1.1) cast in primal weak form yields a variational problem in the Hilbert space $\boldsymbol{H}(\operatorname{curl} ; \Omega)$ of $\boldsymbol{L}^{2}(\Omega)$-vector fields, whose curl is square integrable: Find $\mathbf{u} \in \boldsymbol{H}_{0}(\operatorname{curl} ; \Omega)$ such that for all $\mathbf{q} \in \boldsymbol{H}_{0}(\operatorname{curl} ; \Omega)$

$$
(\chi \operatorname{curl} \mathbf{u}, \operatorname{curl} \mathbf{q})_{L^{2}(\Omega)}+(\beta \mathbf{u}, \mathbf{q})_{L^{2}(\Omega)}=(\mathbf{f}, \mathbf{q})_{L^{2}(\Omega)} .
$$

A subscript 0 indicates that vanishing tangential traces on $\partial \Omega$ are imposed on the fields. For $\beta$ uniformly positive a.e. in $\Omega$ the Lax-Milgram lemma guarantees existence and uniqueness of a solution of (1.2). If $\beta=0$ on sets of positive measure, we can still expect a unique solution for curl $\mathbf{u}$.

It is now generally accepted that an appropriate finite element discretization of (1.2) should rely on genuine $\boldsymbol{H}(\mathbf{c u r l} ; \Omega)$-conforming schemes that merely enforce tangential continuity across interelement boundaries 12, 14, 1]. For simplicial meshes $\boldsymbol{H}(\mathbf{c u r l} ; \Omega)$-conforming finite elements of arbitrary polynomial order were first introduced by Nédélec [47, generalizing the lowest order Whitney elements [58]. Similar schemes are also known for other shapes of elements [47, 28]. For all of them, standard a priori error bounds can be established for shape-regular meshes, as was done in [45, 44, 23]. It turns out that standard families of $\boldsymbol{H}(\mathbf{c u r l} ; \Omega)$ conforming finite elements permit us to approximate $\operatorname{curl} \mathbf{u}$ to the same order as $\mathbf{u}$ itself. Ultimately, the discretization of (1.2) by these so-called edge elements leads to a large sparse linear system of equations.

Keeping in mind that the discretized problem (1.2) has to be solved in each time step, one easily appreciates the need for fast iterative solvers. Suitable multigrid algorithms for $\boldsymbol{H}(\mathbf{c u r l} ; \Omega)$-elliptic variational problems discretized by edge elements have been introduced in [36, 4, 40]. Closely related domain decomposition methods are discussed in [56, 31]. However, the theoretical investigations in these articles do not cover the eddy current setting, because the proofs of stability of the multilevel decompositions fail in the presence of re-entrant corners or locally vanishing coefficients. Some approaches rely heavily on duality techniques [4, 40], which are only feasible if powerful lifting theorems for second-order elliptic operators are available. Those merely hold for smooth or convex domains. On the contrary, the techniques in [36] suffer from their dependence on Helmholtz-decompositions whose weakly solenoidal part has to be $\boldsymbol{H}^{1}(\Omega)$-regular. Again, this is tied to the same restrictions on the shape of $\Omega$ as above. However, in numerical experiments the methods perform excellently in situations far beyond the scope of the hitherto existing theory [36, Sect. 6]. 
Thus, the situation much resembles that in the field of classical multigrids for second order elliptic problems before the arrival of variational multigrid theory [51, [59, 17]: Tight regularity assumptions were necessary to prove the convergence of the $V$-cycle [16, 63]. It was then the feat of variational multilevel theory to launch a successful attack on arbitrary domains and even discontinuous coefficients $[18,19,52]$.

In a sense, this paper aims to extend the scope of theoretical results available for established multilevel schemes for $\boldsymbol{H}(\mathbf{c u r l} ; \Omega)$-conforming finite elements, covering, in particular, regular eddy current problems. More precisely, merely the following restrictions on the geometry will be imposed:

(1) The closure of $\Omega_{C}$ is strictly contained in $\Omega$.

(2) Both $\Omega$ and $\Omega_{C}$ are (curvilinear) Lipschitz-polyhedra in the sense of [33].

(3) The complement $\Omega_{e}:=\Omega \backslash \bar{\Omega}_{C}$ is to be connected.

The crucial idea, how to cope with this general situation, is borrowed from recent studies of singularities of solutions of Maxwell's equations [27, 26]. In short, it was shown that the solutions possess Helmholtz-type decompositions into an $\boldsymbol{H}^{1}(\Omega)$ regular part and a singular part that can be represented by a gradient. This insight already had a strong impact on the theory of numerical methods for Maxwell's equations: it was used to establish discrete compactness of higher order edge elements in [46] and as the basis of numerical methods in 10]. This paper puts forth an application to the analysis of multilevel schemes.

A brief outline is as follows: First, a survey of essential properties of $\boldsymbol{H}(\mathbf{c u r l} ; \Omega)$ conforming edge elements is supplied in Section 2. I emphasize that profound understanding of the traits of edge elements is indispensable for the theoretical treatment of the multilevel scheme. In the third section the notion of a stable nodal multilevel splitting is introduced. Its uniform quasi-orthogonality is established in Section 4. Then, I embark on a detailed derivation of the crucial Helmholtz-type decompositions. In the sixth section these are exploited to show the stability of the nodal multilevel decomposition.

\section{Finite element SPACES}

The Galerkin discretization of (1.2) is based on $\boldsymbol{H}_{0}(\mathbf{c u r l} ; \Omega)$-conforming finite elements. To this end we set up a simplicial or hexahedral triangulation $\mathcal{T}_{h}:=\left\{T_{i}\right\}_{i}$ of $\Omega$ consisting of elements $T_{i}$. $\mathcal{T}_{h}$ is assumed to be quasi-uniform with mesh width $h>0$ and shape-regular in the sense of [22, Ch. 3,§3.1]. In this section, both $\Omega$ and $\mathcal{T}_{h}$ should be read as a generic domain and a related triangulation with mesh width $h$.

We recall Nédélec's $\boldsymbol{H}(\mathbf{c u r l} ; \Omega)$-conforming finite elements from [47] and 30. Sect. 5.3]: For a tetrahedron $T$ the corresponding local spaces of order $k, k \in \mathbb{N}$, are given by

$$
\mathcal{N D}_{k}(T):=\left(\mathcal{P}_{k-1}(T)\right)^{3}+\left\{\mathbf{p} \in\left(\mathcal{P}_{k}(T)\right)^{3} ; \mathbf{p}(\mathbf{x}) \cdot \mathbf{x}=0, \forall \mathbf{x} \in T\right\},
$$

where $\mathcal{P}_{k}(T)$ designates the spaces of polynomials of degree $\leq k$ over $T$. If $T$ is a hexahedron, which is aligned with the coordinate axes, we know

$$
\mathcal{N} \mathcal{D}_{k}(T):=Q_{k-1, k, k}(T) \times Q_{k, k-1, k}(T) \times Q_{k, k, k-1}(T),
$$


where $Q_{k_{1}, k_{2}, k_{3}}(T)$ is the space of polynomials of degree $\leq k_{i}$ in the $i$ th coordinate direction, $i=1,2,3$. Similar local spaces can be found for prisms [47, 28] and pyramids [32].

In this presentation I focus on the classical edge elements, also called Whitney-1forms [13, i.e., the lowest order case $k=1$. Then we have $\mathcal{N D}_{1}(T)=$ $\left\{\mathbf{x} \mapsto \mathbf{a}+\mathbf{b} \times \mathbf{x}, \mathbf{a}, \mathbf{b} \in \mathbb{R}^{3}\right\}$. As a technical tool curl-conforming finite elements of the second kind, sometimes called Mur-Nédélec finite elements 48, 25, will be needed. In the lowest order case their local spaces contain all linear (on tetrahedra) or trilinear (on hexahedra) polynomials, respectively. Cast in formulae this means

$$
\widehat{\mathcal{N D}}_{1}(T)=\mathcal{P}_{1}(T) \quad \text { or } \quad \widehat{\mathcal{N D}}_{1}(T)=Q_{1,1,1}(T) .
$$

Then global finite element spaces can be introduced by

$$
\begin{aligned}
& \mathcal{N D}_{1}\left(\Omega ; \mathcal{T}_{h}\right):=\left\{\mathbf{u} \in \boldsymbol{H}(\operatorname{curl} ; \Omega) ; \mathbf{u}_{\mid T} \in \mathcal{N D}_{1}(T) \forall T \in \mathcal{T}_{h}\right\} \\
& \widehat{\mathcal{N D}}_{1}\left(\Omega ; \mathcal{T}_{h}\right):=\left\{\mathbf{u} \in \boldsymbol{H}(\operatorname{curl} ; \Omega) ; \mathbf{u}_{\mid T} \in \widehat{\mathcal{N D}}_{1}(T) \forall T \in \mathcal{T}_{h}\right\}
\end{aligned}
$$

A subscript 0 will label the corresponding finite element subspace $\mathcal{N} \mathcal{D}_{1,0}\left(\Omega ; \mathcal{T}_{h}\right)$ and $\widehat{\mathcal{N D}}_{1,0}\left(\Omega ; \mathcal{T}_{h}\right)$ of $\boldsymbol{H}_{0}(\mathbf{c u r l} ; \Omega)$ satisfying homogeneous Dirichlet boundary conditions. A superscript 0 tags the subspaces $\boldsymbol{H}^{0}(\operatorname{curl} ; \Omega), \mathcal{N D}_{1}^{0}\left(\Omega ; \mathcal{T}_{h}\right)$ and $\widehat{\mathcal{N D}}_{1}^{0}\left(\Omega ; \mathcal{T}_{h}\right)$ that contain only finite element vector fields with vanishing curl, i.e.,

$$
\begin{aligned}
\boldsymbol{H}^{0}(\operatorname{curl} ; \Omega) & :=\{\mathbf{v} \in \boldsymbol{H}(\operatorname{curl} ; \Omega), \operatorname{curl} \mathbf{v}=0\} \\
\mathcal{N D}_{1}^{0}\left(\Omega ; \mathcal{T}_{h}\right) / \widehat{\mathcal{N D}}_{1}^{0}\left(\Omega ; \mathcal{T}_{h}\right) & :=\boldsymbol{H}^{0}(\operatorname{curl} ; \Omega) \cap \mathcal{N D}_{1}\left(\Omega ; \mathcal{T}_{h}\right) / \widehat{\mathcal{N D}}_{1}\left(\Omega ; \mathcal{T}_{h}\right)
\end{aligned}
$$

I point out that the condition that ensures $\boldsymbol{H}(\mathbf{c u r l} ; \Omega)$-conformity is the continuity of tangential components across interelement faces [47, 35. In practice, it is enforced by an appropriate choice of degrees of freedom (d.o.f.). In the lowest order case $k=1$, the sets of d.o.f. are given by weighted path integrals along the oriented edges of the mesh $\mathcal{T}_{h}$ [35, 47]:

$$
\begin{array}{lll}
\text { for } \mathcal{N} \mathcal{D}_{1}\left(\Omega ; \mathcal{T}_{h}\right): & \Xi_{1}\left(\mathcal{T}_{h}\right):= & \left\{\mathbf{u}_{h} \mapsto \int_{e} \mathbf{u}_{h} \cdot d \vec{s}, \quad e \text { edge of } \mathcal{T}_{h}\right\}, \\
\text { for } \widehat{\mathcal{N D}}_{1}\left(\Omega ; \mathcal{T}_{h}\right): & \widehat{\Xi}_{1}\left(\mathcal{T}_{h}\right):=\quad \Xi_{1}\left(\mathcal{T}_{h}\right) \cup\left\{\mathbf{u}_{h} \mapsto \int_{e} \pi_{e} \mathbf{u}_{h} \cdot d \vec{s}, \quad e \text { edge of } \mathcal{T}_{h}\right\},
\end{array}
$$

where $\pi_{e}$ is linear along the edge $e$ assuming the value +1 at one endpoint and -1 at the other. In the case of $\mathcal{N} \mathcal{D}_{1,0}\left(\Omega ; \mathcal{T}_{h}\right)$ and $\widehat{\mathcal{N D}}_{1,0}\left(\Omega ; \mathcal{T}_{h}\right)$, one has to skip the edges on $\partial \Omega$.

All degrees of freedom remain invariant under a suitable covariant transformation of vector fields, which also commutes with the application of the curl-operator (see [47, 35]). Firstly, this immediately supplies edge elements for curvilinear elements, which we need to deal with smooth curved parts of the boundary. Secondly, edge elements of any kind become affine families of finite elements in the sense of 22 . Consequently, affine equivalence techniques are an all-important tool in the investigation of properties of edge elements. A first application yields the $L^{2}$-stability of nodal bases. Remember that those are defined as sets of localized finite element functions dual to $\Xi_{1}\left(\mathcal{T}_{h}\right)$ and $\widehat{\Xi}_{1}\left(\mathcal{T}_{h}\right)$, respectively. 
Lemma 2.1. Denote by $\left\{\mathbf{b}_{\kappa}\right\}, \kappa \in \Xi_{1}\left(\mathcal{T}_{h}\right)$, and $\hat{\mathbf{b}}_{\kappa}, \kappa \in \widehat{\Xi}_{1}\left(\mathcal{T}_{h}\right)$, the nodal bases of $\mathcal{N D}_{1}\left(\Omega ; \mathcal{T}_{h}\right)$ and $\widehat{\mathcal{N D}}_{1}\left(\Omega ; \mathcal{T}_{h}\right)$, respectively. Then,

$$
\left\|\mathbf{u}_{h}\right\|_{\boldsymbol{L}^{2}(\Omega)}^{2} \asymp \sum_{\kappa \in \Xi_{1}\left(\mathcal{T}_{h}\right)} \kappa\left(\mathbf{u}_{h}\right)^{2}\left\|\mathbf{b}_{\kappa}\right\|_{\boldsymbol{L}^{2}(\Omega)}^{2}
$$

and

$$
\left\|\hat{\mathbf{u}}_{h}\right\|_{\boldsymbol{L}^{2}(\Omega)}^{2} \asymp \sum_{\kappa \in \hat{\Xi}_{1}\left(\mathcal{T}_{h}\right)} \kappa\left(\hat{\mathbf{u}}_{h}\right)^{2}\left\|\hat{\mathbf{b}}_{\kappa}\right\|_{\boldsymbol{L}^{2}(\Omega)}^{2} .
$$

for all for all $\mathbf{u}_{h} \in \mathcal{N D}_{1}\left(\Omega ; \mathcal{T}_{h}\right), \hat{\mathbf{u}}_{h} \in \widehat{\mathcal{N D}}_{1}\left(\Omega ; \mathcal{T}_{h}\right)$.

Taking the cue from [59], I adopt the notation $\asymp$ to express equivalence up to constants that, unless stated otherwise, only depend on the geometry, i.e., $\Omega$ and $\Omega_{C}$, and the shape regularity of the triangulations. Estimates up to such constants will be marked by $\lesssim$ and $\gtrsim$, respectively. The value of the constants may vary between different occurrences of these symbols.

For later use we cite some properties of the nodal basis functions

$$
\left\|\mathbf{b}_{\kappa}\right\|_{L^{2}(\Omega)}^{2} \asymp h, \quad\left\|\mathbf{b}_{\kappa}\right\|_{L^{2}(\Omega)} \lesssim h\left\|\operatorname{curl} \mathbf{b}_{\kappa}\right\|_{L^{2}(\Omega)}
$$

for all $\kappa \in \Xi_{1}\left(\mathcal{T}_{h}\right)$. The same relationships hold for all $\hat{\mathbf{b}}_{\kappa}$. It is also worth mentioning that the support of $\mathbf{b}_{\kappa}$ is confined to those elements of $\mathcal{T}_{h}$ that are adjacent to the edge to which $\kappa$ belongs.

Now, given the degrees of freedom, the nodal projections (nodal interpolation operators) $\Pi_{h}$ and $\widehat{\Pi}_{h}$ onto the finite element spaces $\mathcal{N} \mathcal{D}_{1}\left(\Omega ; \mathcal{T}_{h}\right)$ and $\widehat{\mathcal{N} \mathcal{D}_{1}}\left(\Omega ; \mathcal{T}_{h}\right)$ can be introduced as in 22 . For smooth vector fields they are straightforward, but $\widehat{\Pi}_{h}$ fails to be defined on the entire function space $\boldsymbol{H}(\operatorname{curl} ; \Omega)$ and even $\boldsymbol{H}^{1}(\Omega)$, because the d.o.f. in $\widehat{\Xi}_{1}\left(\mathcal{T}_{h}\right)$ fail to provide continuous linear functionals on these spaces. A slightly enhanced smoothness of the argument function is required, because the integrals along edges are continuous functionals only for vector fields, which locally belong to the space $\mathbf{W}_{p}(T)$ for $p>2$ and all $T \in \mathcal{T}_{h}$ [3, Lemma 4.7]. $\mathbf{W}_{p}(T)$ is given by

$$
\mathbf{W}_{p}(T):=\left\{\mathbf{w} \in\left(L^{p}(T)\right)^{3}, \mathbf{c u r l} \mathbf{w} \in\left(L^{p}(T)\right)^{3}, \mathbf{w} \times \mathbf{n} \in\left(L^{p}(\partial T)\right)^{2}\right\} .
$$

Edge elements can be viewed as discrete differential forms [15]. As such they possess discrete potentials in spaces of $H^{1}(\Omega)$-conforming finite element functions [35, Thm. 18], if the domain covered by the triangulation $\mathcal{T}_{h}$ is simply connected. For $\mathcal{N} \mathcal{D}_{1}\left(\Omega ; \mathcal{T}_{h}\right)$ the discrete potential space agrees with the space $\mathcal{S}_{1}\left(\Omega ; \mathcal{T}_{h}\right)$ of piece-wise linear, globally continuous functions on $\mathcal{T}_{h}$. In the case of $\widehat{\mathcal{N} \mathcal{D}_{1}}\left(\Omega ; \mathcal{T}_{h}\right)$ the potential space is $\mathcal{S}_{2}\left(\Omega ; \mathcal{T}_{h}\right)$, which contains the continuous, piece-wise quadratic finite element functions. In short, we have for simply connected $\Omega$

$$
\mathcal{N} \mathcal{D}_{1}^{0}\left(\Omega ; \mathcal{T}_{h}\right)=\operatorname{grad} \mathcal{S}_{1}\left(\Omega ; \mathcal{T}_{h}\right) \quad \text { and } \quad \widehat{\mathcal{N D}}_{1}^{0}\left(\Omega ; \mathcal{T}_{h}\right)=\operatorname{grad} \mathcal{S}_{2}\left(\Omega ; \mathcal{T}_{h}\right)
$$

I point out that the potentials can be chosen in $H_{0}^{1}(\Omega)$ if the edge element functions satisfy homogeneous boundary conditions and $\partial \Omega$ is connected. The symbol $\mathcal{S}_{k}\left(\Omega ; \mathcal{T}_{h}\right)$ will be used for vector fields, whose Cartesian components belong to $\mathcal{S}_{k}\left(\Omega ; \mathcal{T}_{h}\right)$. The following embeddings are trivial but fundamental in our investigations:

$$
\mathcal{N D}_{1}\left(\Omega ; \mathcal{T}_{h}\right) \subset \widehat{\mathcal{N D}}_{1}\left(\Omega ; \mathcal{T}_{h}\right) \quad \text { and } \quad \mathcal{S}_{1}\left(\Omega ; \mathcal{T}_{h}\right) \subset \widehat{\mathcal{N D}}_{1}\left(\Omega ; \mathcal{T}_{h}\right)
$$


It is their unique algebraic features that render the nodal interpolation operators indispensable. They are the only known locally defined projection operators that satisfy a so-called commuting diagram property (cf. Remark 3.2 in [29]). Recall that $\operatorname{curl} \mathcal{N D}_{1}\left(\Omega ; \mathcal{T}_{h}\right) \subset \mathcal{R} \mathcal{T}_{0}\left(\Omega ; \mathcal{T}_{h}\right)$ and $\operatorname{curl} \widehat{\mathcal{N D}}_{1}\left(\Omega ; \mathcal{T}_{h}\right) \subset \mathcal{R} \mathcal{T}_{0}\left(\Omega ; \mathcal{T}_{h}\right)$, where $\boldsymbol{R} \mathcal{T}_{0}\left(\Omega ; \mathcal{T}_{h}\right)$ is the lowest-order $\boldsymbol{H}(\operatorname{div} ; \Omega)$-conforming Raviart-Thomas finite element space [21, III.3.1]. When writing $J_{h}$ for the canonical interpolation operators onto $\mathcal{R} \mathcal{T}_{0}\left(\Omega ; \mathcal{T}_{h}\right)$, it turns out [35, Thm. 13] that for all $\mathbf{w} \in \mathbf{W}_{p}\left(\mathcal{T}_{h}\right):=$ $\left\{\mathbf{v} \in \boldsymbol{H}(\operatorname{curl} ; \Omega), \mathbf{v}_{\mid T} \in \mathbf{W}_{p}(T) \forall T \in \mathcal{T}_{h}\right\}, p>2$,

$$
\left(J_{h} \circ \operatorname{curl}\right) \mathbf{w}=\left(\operatorname{curl} \circ \Pi_{h}\right) \mathbf{w} \quad \text { and } \quad\left(J_{h} \circ \operatorname{curl}\right) \mathbf{w}=\left(\operatorname{curl} \circ \widehat{\Pi}_{h}\right) \mathbf{w} .
$$

Another commuting diagram property involves the nodal interpolation operators $I_{h}$ and $\widehat{I}_{h}$ for $\mathcal{S}_{1}\left(\Omega ; \mathcal{T}_{h}\right)$ and $\mathcal{S}_{2}\left(\Omega ; \mathcal{T}_{h}\right)$, respectively. It claims that

$$
\left(\operatorname{grad} \circ I_{h}\right) \phi=\left(\Pi_{h} \circ \operatorname{grad}\right) \phi \quad \text { and }\left(\operatorname{grad} \circ \widehat{I}_{h}\right) \phi=\left(\widehat{\Pi}_{h} \circ \operatorname{grad}\right) \phi
$$

for all continuous functions $\phi$ in $H^{1}(\Omega)$. We may use (2.6) along with affine equivalence techniques to establish the following stable splitting.

Lemma 2.2. The space $\widehat{\mathcal{N D}}_{1}\left(\Omega ; \mathcal{T}_{h}\right)$ is the $\boldsymbol{L}^{2}(\Omega)$-stable direct sum of $\mathcal{N D}_{1}\left(\Omega ; \mathcal{T}_{h}\right)$ and $\operatorname{grad} \hat{\mathcal{S}}_{2}\left(\Omega ; \mathcal{T}_{h}\right)$, where $\hat{\mathcal{S}}_{2}\left(\Omega ; \mathcal{T}_{h}\right)=\left(I d-I_{h}\right) \mathcal{S}_{2}\left(\Omega ; \mathcal{T}_{h}\right)$ is the quadratic hierarchical surplus. This means that for all $\hat{\mathbf{u}}_{h} \in \widehat{\mathcal{N D}}_{1}\left(\Omega ; \mathcal{T}_{h}\right)$ there are unique $\mathbf{u}_{h} \in \mathcal{N D}_{1}\left(\Omega ; \mathcal{T}_{h}\right)$ and $\hat{\phi}_{h} \in \hat{\mathcal{S}}_{2}\left(\Omega ; \mathcal{T}_{h}\right)$ such that

$$
\hat{\mathbf{u}}_{h}=\mathbf{u}_{h}+\operatorname{grad} \hat{\phi}_{h} \quad \text { and } \quad\left\|\mathbf{u}_{h}\right\|_{\boldsymbol{L}^{2}(\Omega)}+\left\|\operatorname{grad} \hat{\phi}_{h}\right\|_{\boldsymbol{L}^{2}(\Omega)} \lesssim\left\|\hat{\mathbf{u}}_{h}\right\|_{\boldsymbol{L}^{2}(\Omega)} .
$$

As for technical tools, I have to resort to the boundary compliant quasi-interpolation operators according to Scott and Zhang [54]: $Q_{h}: H^{1}(\Omega) \mapsto \mathcal{S}_{1}\left(\Omega ; \mathcal{T}_{h}\right)$ and $\widehat{Q}_{h}: H^{1}(\Omega) \mapsto \mathcal{S}_{2}\left(\Omega ; \mathcal{T}_{h}\right)$. They furnish projections with the continuity and approximations properties

$$
\left\|Q_{h} \phi\right\|_{H^{1}(\Omega)} \lesssim\|\phi\|_{H^{1}(\Omega)}, \quad\left\|\phi-Q_{h} \phi\right\|_{L^{2}(\Omega)} \lesssim h\|\phi\|_{H^{1}(\Omega)} \quad \forall \phi \in H^{1}(\Omega) .
$$

In addition, these projectors leave boundary values invariant if those are traces of finite element functions in the range of the projectors.

Finally, I will examine the traces of finite element vector fields. First note that the mesh $\mathcal{T}_{h}$ induces a shape-regular, quasi-uniform triangulation $\mathcal{T}_{h}^{\partial}$ of the surface $\partial \Omega$ of $\Omega$. We can then view the tangential trace $\mathbf{u}_{h} \times \mathbf{n}_{\mid \partial \Omega}$ of an edge element vector field $\mathbf{u}_{h}$ as a piece-wise polynomial $2 \mathrm{D}$ vector field on $\partial \Omega$. More precisely, a closer scrutiny reveals [35]:

Lemma 2.3. For $\boldsymbol{\xi} \in \mathcal{N}_{\mathcal{D}_{1}}\left(\Omega ; \mathcal{T}_{h}\right)$, we have $\boldsymbol{\xi}_{h} \times \mathbf{n}_{\mid \partial \Omega} \in \mathcal{R} \mathcal{T}_{0}\left(\Omega ; \mathcal{T}_{h}^{\partial}\right)$, where $\boldsymbol{R} \mathcal{T}_{0}\left(\Omega ; \mathcal{T}_{h}^{\partial}\right)$ is the $\boldsymbol{H}\left(\operatorname{div}_{\partial} ; \partial \Omega\right)$-conforming space of $2 D$ Raviart-Thomas finite element functions of lowest order as introduced in [53, 21]. Similarly, the tangential trace of $\widehat{\mathcal{N D}}_{1}\left(\Omega ; \mathcal{T}_{h}\right)$ agrees with the space $\widehat{\mathcal{R T}}_{0}\left(\Omega ; \mathcal{T}_{h}^{2}\right)$ of two-dimensional BDM elements [20, 21] on the surface $\partial \Omega$.

In addition, we observe that the degrees of freedom in $\Xi_{1}\left(\mathcal{T}_{h}\right)$, which belong to edges on the boundary $\partial \Omega$, agree with the canonical degrees of freedom of $\mathcal{R} \mathcal{T}_{0}\left(\Omega ; \mathcal{T}_{h}^{\partial}\right)$. The same is true for $\widehat{\Xi}_{1}\left(\mathcal{T}_{h}\right)$ and $\widehat{\mathcal{R T}}_{0}\left(\Omega ; \mathcal{T}_{h}^{\partial}\right)$. Thus, we get from an $L^{2}$-stability estimate for Raviart-Thomas elements in two dimensions [39]

$$
\left\|\mathbf{u}_{h} \times \mathbf{n}\right\|_{\boldsymbol{L}^{2}(\partial \Omega)}^{2} \asymp \sum_{\kappa} \kappa\left(\mathbf{u}_{h}\right)^{2},
$$


where the sum covers all degrees of freedom in $\Xi_{1}\left(\mathcal{T}_{h}\right)$ or $\widehat{\Xi}_{1}\left(\mathcal{T}_{h}\right)$ that lie on the boundary of $\Omega$.

\section{NodAl multilevel SPlitting}

The crucial variational problem (1.2) involves the symmetric, positive semidefinite bilinear form $a: \boldsymbol{H}_{0}(\operatorname{curl} ; \Omega) \times \boldsymbol{H}_{0}(\mathbf{c u r l} ; \Omega) \mapsto \mathbb{R}$ given by

$$
a(\mathbf{u}, \mathbf{v}):=\left(\chi_{0} \operatorname{curl} \mathbf{u}, \mathbf{c u r l} \mathbf{v}\right)_{\boldsymbol{L}^{2}(\Omega)}+\left(\beta_{0} \mathbf{u}, \mathbf{v}\right)_{\mathbf{L}^{2}\left(\Omega_{C}\right)}
$$

On top of that, the discretization relies on a conforming Galerkin scheme. This makes the problem amenable to the variational theory of multilevel Schwarz methods. In this framework standard multigrid methods and multilevel preconditioners arise from a nodal multilevel decomposition of the underlying finite element space $\mathcal{N} \mathcal{D}_{1,0}\left(\Omega ; \mathcal{T}_{h}\right)$

The background is that a discrete variational problem based on the bilinear form $a(\cdot, \cdot)$ can be viewed as a minimization problem for a quadratic energy functional. Writing the trial space as a sum of subspaces, successive minimization of the energy functional (the multiplicative Schwarz method) in the direction of these subspaces leads to an iterative scheme, which is closely related to von Neumann's method of alternating projections [57] (see [61, Sect. 3,4]). Parallel minimization (the additive Schwarz method) gives rise to preconditioners. There is a huge body of literature on the theory and practical implementation of (multilevel) Schwarz methods starting from 43 . I merely refer to the survey papers [59, 60, 63 and the books [17, 55. for comprehensive presentations. I stress that the subspace decomposition completely determines the additive Schwarz method. In the case of the multiplicative variant, we additionally have to specify an ordering of the subspaces. Tersely speaking, a complete theoretical analysis of the schemes only needs to study the underlying subspace decomposition. Of course, efficient implementation is still a challenge, but this is outside the scope of the present paper. For information about the algorithmic aspects of the multilevel methods discussed in this paper, the reader is referred to [36, Sect. 6], 6, Sect. 7.3], 37, Sect. 7], and [7].

To fix the multilevel setting, we assume that we have a nested sequence $\mathcal{T}_{0} \prec$ $\mathcal{T}_{1} \prec \ldots, \prec \mathcal{T}_{L}, L \in \mathbb{N}$, of quasiunform triangulations at our disposal, which has been created by regular refinement of some initial mesh $\mathcal{T}_{0}$ (coarse grid). Its mesh width $h_{0}$ is supposed to be 1 , which can always be achieved by scaling. The coarse grid has to provide a full description of the geometry in the sense that the conductor $\Omega_{C}$ is completely resolved by $\mathcal{T}_{0}$. Nesting ensures that this is satisfied for all other meshes $\mathcal{T}_{l}$, too. Thus we can single out two sequences of subgrids

$$
\mathcal{T}_{l}^{C}:=\left\{T \in \mathcal{T}_{l}, T \subset \Omega_{C}\right\}, \quad \mathcal{T}_{l}^{e}:=\left\{T \in \mathcal{T}_{l}, T \subset \Omega_{e}\right\}, \quad l=0, \ldots, L,
$$

and restrictions of the meshes to boundaries and interfaces

$$
\mathcal{T}_{l}^{\Gamma}:=\mathcal{T}_{l} \text { on } \partial \Omega_{C}, \quad \mathcal{T}_{l}^{\partial}:=\mathcal{T}_{l} \text { on } \partial \Omega, \quad l=0, \ldots, L
$$

Refinement strategies that guarantee uniform shape regularity of the $\mathcal{T}_{l}, l=0, \ldots, L$, have been introduced, for instance, in [8] 5]. The mesh widths $h_{l}, l=0, \ldots, L$, are expected to decrease in geometric progression so that $h_{l} \asymp 2^{-l}$. With each level $l$ we associate the finite element space $\mathcal{N} \mathcal{D}_{1,0}\left(\Omega ; \mathcal{T}_{l}\right)$. These spaces are contained in each other, i.e., $\mathcal{N D}_{1,0}\left(\Omega ; \mathcal{T}_{l-1}\right) \subset \mathcal{N} \mathcal{D}_{1,0}\left(\Omega ; \mathcal{T}_{l}\right), l=1, \ldots, L$. 
The multigrid method from [36, 40] relies on the following nodal multilevel decompositions of both the edge element space and the discrete potential space

$$
\begin{aligned}
\mathcal{N D}_{1,0}\left(\Omega ; \mathcal{T}_{L}\right)=\mathcal{N D}_{1,0}\left(\Omega ; \mathcal{T}_{0}\right) & +\sum_{l=1}^{L} \sum_{\kappa \in \Xi_{1}\left(\mathcal{T}_{l}\right)} \operatorname{Span}\left\{\mathbf{b}_{\kappa}\right\} \\
& +\sum_{l=1}^{L} \sum_{\mathbf{x} \in \mathcal{N}_{l}} \operatorname{Span}\left\{\operatorname{grad} \psi_{\mathbf{x}}\right\}
\end{aligned}
$$

where $\{\mathbf{b}\}_{\kappa}$ is the nodal basis of $\mathcal{N D}_{1,0}\left(\Omega ; \mathcal{T}_{l}\right)$ and $\mathcal{N}_{l}$ designates the set of interior vertices of $\mathcal{T}_{l}$. Further, $\left\{\psi_{\mathbf{x}}\right\}_{\mathbf{x} \in \mathcal{N}_{l}}$ is the nodal basis of the space $\mathcal{S}_{1}\left(\Omega ; \mathcal{T}_{l}\right)$ of linear Lagrangian finite element functions. The rationale for choosing the multilevel splitting (3.1) is thoroughly explained in [36, Sect. 3] and [40, Sect. 3].

From [59, Sect. 5] we learn that it is essential that the decomposition (3.1) is sufficiently stable with respect to the energy semi-norm $\|\cdot\|_{\mathfrak{E}: \Omega}:=a(\cdot, \cdot)^{\frac{1}{2}}$. At first glance, it may seem that the theory of [59] does not apply, because $a(\cdot, \cdot)$ has a nontrivial kernel. There is a simple remedy, however, and it boils down to switching to factor spaces modulo $\operatorname{Ker}(a)$ : Let us write $\widetilde{\mathcal{N D}}_{1,0}\left(\Omega ; \mathcal{T}_{L}\right):=\mathcal{N}_{\mathcal{D}_{1,0}}\left(\Omega ; \mathcal{T}_{L}\right) / \operatorname{Ker}(a)$ and observe that $a$ spawns an inner product $\widetilde{a}$ on $\widehat{\mathcal{N D}}_{1,0}\left(\Omega ; \mathcal{T}_{L}\right)$. Thus, $\widetilde{\mathcal{N D}}_{1,0}\left(\Omega ; \mathcal{T}_{L}\right)$ equipped with $\widetilde{a}$ provides just the right setting for the abstract theory of [59].

Throughout, operators in the factor space will be tagged by ${ }^{\sim}$. In particular, I denote by $\widetilde{A}_{L}: \widetilde{\mathcal{N D}}_{1,0}\left(\Omega ; \mathcal{T}_{L}\right) \mapsto \widetilde{\mathcal{N D}}_{1,0}\left(\Omega ; \mathcal{T}_{L}\right)^{\prime}$ the s.p.d. operator associated with $\widetilde{a}$. The $\widetilde{a}$-orthogonal projections $\widetilde{P}_{\kappa}: \widetilde{\mathcal{N D}}_{1,0}\left(\Omega ; \mathcal{T}_{L}\right) \mapsto \operatorname{Span}\left\{\mathbf{b}_{\kappa}\right\} / \operatorname{Ker}(a)$, $\kappa \in \Xi_{1}\left(\mathcal{T}_{l}\right), l=1, \ldots, L$, are given, in terms of representatives, by

$$
P_{\kappa} \mathbf{u}_{h}:=\frac{a\left(\mathbf{u}_{h}, \mathbf{b}_{\kappa}\right)}{a\left(\mathbf{b}_{\kappa}, \mathbf{b}_{\kappa}\right)} \cdot \mathbf{b}_{\kappa} .
$$

Obviously, they are well defined on the factor space. Moreover, (3.2) also makes sense on the actual finite element space, because $a\left(\mathbf{b}_{\kappa}, \mathbf{b}_{\kappa}\right) \neq 0$ for all $\kappa \in \Xi_{1}\left(\mathcal{T}_{l}\right)$, $l=1, \ldots, L$. This greatly benefits the implementation of the method, because one does not have to handle factor spaces.

The family $\widetilde{P}_{\mathbf{x}}: \widetilde{\mathcal{N D}}_{1,0}\left(\Omega ; \mathcal{T}_{L}\right) \mapsto \operatorname{Span}\left\{\operatorname{grad} \psi_{\mathbf{x}}\right\} / \operatorname{Ker}(a), \mathbf{x} \in \mathcal{N}_{l}^{C}, l=1, \ldots, L$, of $\tilde{a}$-orthogonal projections is defined in terms of representatives by

$$
P_{\mathbf{x}} \mathbf{u}_{h}=\frac{a\left(\mathbf{u}_{h}, \operatorname{grad} \psi_{\mathbf{x}}\right)}{a\left(\operatorname{grad} \psi_{\mathbf{x}}, \operatorname{grad} \psi_{\mathbf{x}}\right)} \cdot \operatorname{grad} \psi_{\mathbf{x}}
$$

Again, these projections can be considered in the real finite element space. Note that the range of $\mathbf{x}$ has to be restricted to vertices of $\mathcal{T}_{l}^{C}$, which form the set $\mathcal{N}_{l}^{C}$. Otherwise ill-defined terms "0 0 " would occur in (3.3).

Then the approximate inverse $\widetilde{B}_{L}^{\text {add }}: \widetilde{\mathcal{N D}}_{1,0}\left(\Omega ; \mathcal{T}_{L}\right)^{\prime} \mapsto \widetilde{\mathcal{N D}}_{1,0}\left(\Omega ; \mathcal{T}_{L}\right)$ of $\widetilde{A}_{L}$ provided by the additive Schwarz method related to (3.1) is given by (cf. [59] Sect. 3.2])

$$
\widetilde{B}_{L}^{\text {add }} \widetilde{A}_{L}=\widetilde{P}_{0}+\sum_{l=1}^{L}\left(\sum_{\kappa \in \Xi_{1}\left(\mathcal{T}_{l}\right)} \widetilde{P}_{\kappa}+\sum_{\mathbf{x} \in \mathcal{N}_{l}^{C}} \widetilde{P}_{\mathbf{x}}\right)
$$


where $\widetilde{P}_{0}$ is the $\widetilde{a}$-orthogonal projection onto $\widetilde{\mathcal{N D}}_{1,0}\left(\Omega ; \mathcal{T}_{0}\right)$. In order to describe the corresponding operator $\widetilde{B}_{L}^{\text {mul }}: \widetilde{\mathcal{N D}}_{1,0}\left(\Omega ; \mathcal{T}_{L}\right)^{\prime} \mapsto \widetilde{\mathcal{N D}}_{1,0}\left(\Omega ; \mathcal{T}_{L}\right)$ arising from the symmetric multiplicative Schwarz method (multigrid) we introduce

$$
\widetilde{R}_{l}:=\prod_{\kappa \in \Xi_{1}\left(\mathcal{T}_{l}\right)}\left(\widetilde{I d}-\widetilde{P}_{\kappa}\right), \quad \widetilde{S}_{l}:=\prod_{\mathbf{x} \in \mathcal{N}_{l}^{C}}\left(\widetilde{I d}-\widetilde{P}_{\mathbf{x}}\right), \quad \widetilde{T}_{l}:=\widetilde{S}_{l} \widetilde{R}_{l} .
$$

Then, we obtain (cf. [59, Sect. 3.3])

$$
\widetilde{I d}-\widetilde{B}_{L}^{\mathrm{mul}} \widetilde{A}_{L}=\widetilde{T}_{L}^{*} \widetilde{T}_{L-1}^{*} \cdots \cdot \widetilde{T}_{1}^{*} \widetilde{T}_{0} \widetilde{T}_{1} \cdots \widetilde{T}_{L-1} \widetilde{T}_{L},
$$

where $\widetilde{T}_{0}:=\widetilde{I d}-\widetilde{P}_{0}$ and $*$ designates $\widetilde{a}$-adjoints. I point out that (3.5) supplies the error propagation operator of the multigrid iteration with symmetric Gauß-Seidel smoother. It is also worth noting that both $\widetilde{B}_{L}^{\text {add }} \widetilde{A}_{L}$ and $\widetilde{B}_{L}^{\text {mul }} \widetilde{A}_{L}$ are $\widetilde{a}$-self-adjoint and positive definite, and, thus, possess a positive real spectrum.

According to [59, Sect. 5], stability of a subspace decomposition can be gauged by means of two estimates. The first is a strengthened Cauchy-Schwarz inequality that makes a statement about the quasi-orthogonality of the subspaces involved in the decomposition. It will be stated and proved in the next section. The second may be called a stability estimate. Formally writing $\left\{\mathcal{V}_{j}\right\}_{j}$ for the set of subspaces in (3.1), it can be stated as

$$
\inf \left\{\sum_{j}\left\|\mathbf{v}_{j}\right\|_{\mathfrak{E} ; \Omega}^{2} ; \sum_{j} \mathbf{v}_{j}=\mathbf{u}_{h}, \mathbf{v}_{j} \in \mathcal{V}_{j}\right\} \lesssim C_{\text {stab }}\left\|\mathbf{u}_{h}\right\|_{\mathfrak{E} ; \Omega}^{2}
$$

for all $\mathbf{u}_{h} \in \mathcal{N}_{\mathcal{D}_{1,0}}\left(\Omega ; \mathcal{T}_{L}\right)$. Here, $C_{\text {stab }}>0$ may be a function of the ratio $\chi_{0} / \beta_{0}$. The objective of the remainder of this paper, except for the next section, is to establish (3.6) and, in particular, to make sure that the norm equivalence will hold independently of the depth $L$ of refinement.

\section{Quasi-OrThogonality}

To prove a strengthened Cauchy-Schwarz inequality, I pursue a strategy analogous to what has been done in [11, 62] and [59, Sect. 7.1.1] for the standard $H^{1}$ conforming case. The proof is conducted parallel to that detailed in Sect. 6 of [34 for the case of Raviart-Thomas elements. To begin with, elementary combinatorial arguments verify the following "coloring lemma".

Lemma 4.1. The vertex sets $\mathcal{N}_{l}$ and edge sets $\mathcal{E}_{l}$ of $\mathcal{T}_{l}, l=0, \ldots, L$, can be partitioned into subsets $\mathcal{N}_{l}=\mathrm{V}_{l}^{1} \cup \cdots \cup \mathrm{V}_{l}^{P}, \mathcal{E}_{l}=\mathrm{E}_{l}^{1} \cup \cdots \cup \mathrm{E}_{l}^{K}, P, K \in \mathbb{N}$, independent of $l$, such that any $T \in \mathcal{T}_{l}$ possesses only vertices/edges from different subsets.

As the nodal basis functions of $\mathcal{N D}_{1,0}\left(\Omega ; \mathcal{T}_{l}\right)$ and $\mathcal{S}_{1,0}\left(\Omega ; \mathcal{T}_{l}\right), l=0, \ldots, L$, associated with edges and vertices in $\mathrm{E}_{l}^{i}$ and $\mathrm{V}_{l}^{j}$, respectively, have nonoverlapping supports, they are $a(\cdot, \cdot)$-orthogonal. Hence, the stability properties of (3.1) and those of the splitting

$$
\begin{aligned}
& \mathcal{N D}_{1,0}\left(\Omega ; \mathcal{T}_{L}\right)=\mathcal{N}_{\mathcal{D}_{1,0}}\left(\Omega ; \mathcal{T}_{0}\right) \\
& \quad+\sum_{l=1}^{L}(\sum_{i=1}^{K} \underbrace{\operatorname{Span}\left\{\mathbf{b}_{\kappa}, \kappa \in \Xi_{1}^{i}\left(\mathcal{T}_{l}\right)\right\}}_{=: \mathcal{D}_{l}^{i}}+\sum_{j=1}^{P} \operatorname{grad} \underbrace{\operatorname{Span}\left\{\psi_{\mathbf{x}}, \mathbf{x} \in \mathrm{V}_{l}^{j}\right\}}_{=: \mathcal{C}_{l}^{j}}),
\end{aligned}
$$


with $\Xi_{1}^{i}\left(\mathcal{T}_{l}\right)$ standing for those d.o.f. of $\Xi_{1}\left(\mathcal{T}_{l}\right)$ that belong to edges in $\mathrm{E}_{l}^{i}, i=$ $1, \ldots, K$, are exactly the same.

Lemma 4.2. For all $\mathbf{v}_{m} \in \mathcal{N D}_{1,0}\left(\Omega ; \mathcal{T}_{m}\right)$ and $\mathbf{d}_{l} \in \mathcal{D}_{l}^{i}, 0 \leq m \leq l \leq L, i=$ $1, \ldots, K$, it holds that

$$
\begin{aligned}
\left(\operatorname{curl}_{m}, \operatorname{curl}_{l}\right)_{\boldsymbol{L}^{2}(\Omega)} & \lesssim h_{l} / h_{m}\left\|\operatorname{curl} \mathbf{v}_{m}\right\|_{\boldsymbol{L}^{2}(\Omega)}\left\|\operatorname{curl} \mathbf{d}_{l}\right\|_{\boldsymbol{L}^{2}(\Omega)}, \\
\left(\mathbf{v}_{m}, \mathbf{d}_{l}\right)_{\boldsymbol{L}^{2}\left(\Omega_{C}\right)} & \lesssim h_{l}\left\|\mathbf{v}_{m}\right\|_{\boldsymbol{L}^{2}\left(\Omega_{C}\right)}\left\|\operatorname{curl} \mathbf{d}_{l}\right\|_{\boldsymbol{L}^{2}\left(\Omega_{C}\right)} .
\end{aligned}
$$

Proof. Pick any $T \in \mathcal{T}_{m}$ and observe that $\operatorname{curl} \mathbf{v}_{m \mid T}$ is constant. Then, by Green's formula,

$$
\begin{aligned}
\int_{T} \operatorname{curl}_{m} \cdot \operatorname{curl} \mathbf{d}_{l} d \mathbf{x} & =\operatorname{curl}_{m \mid T} \int_{\partial T} \mathbf{d}_{l} \times \mathbf{n} d S \\
& =\int_{\Sigma_{T}} \operatorname{curl}_{m} \cdot \operatorname{curl} \mathbf{d}_{l} d \mathbf{x} \\
& \leq\left\|\operatorname{curl}_{m}\right\|_{\mathbf{L}^{2}\left(\Sigma_{T}\right)}\left\|\operatorname{curl} \mathbf{d}_{l}\right\|_{\boldsymbol{L}^{2}\left(\Sigma_{T}\right)} \\
& \leq \frac{\left|\Sigma_{T}\right|}{|T|}\left\|\operatorname{curl}_{m}\right\|_{\boldsymbol{L}^{2}(T)}\left\|\operatorname{curl} \mathbf{d}_{l}\right\|_{\boldsymbol{L}^{2}(T)} \\
& \lesssim h_{l} h_{m}^{-1}\left\|\operatorname{curl} \mathbf{v}_{m}\right\|_{\boldsymbol{L}^{2}(T)}\left\|\operatorname{curl}_{l}\right\|_{\boldsymbol{L}^{2}(T)},
\end{aligned}
$$

where $\Sigma_{T}:=\left\{\widetilde{T} \in \mathcal{T}_{l}, \widetilde{T} \subset T, \partial \widetilde{T} \cap \partial T \neq \emptyset\right\}$ (cf. the proof of Lemma 7.1 in [59). Summing over all $T \in \mathcal{T}_{m}$ and applying the Cauchy-Schwarz inequality gives the first estimate. The second follows immediately from (2.1) and the definition of $\mathcal{D}_{l}^{i}$.

Lemma 4.3. For all $\mathbf{v}_{m} \in \mathcal{N}_{\mathcal{D}_{1,0}}\left(\Omega ; \mathcal{T}_{m}\right)$ and $\phi_{l} \in \mathcal{C}_{l}^{j}, 0 \leq m \leq l \leq L, j=$ $1, \ldots, P$, we have

$$
\left(\mathbf{v}_{m}, \operatorname{grad} \phi_{l}\right)_{\boldsymbol{L}^{2}\left(\Omega_{C}\right)} \lesssim h_{l} h_{m}^{-1}\left\|\mathbf{v}_{m}\right\|_{\boldsymbol{L}^{2}\left(\Omega_{C}\right)}\left\|\operatorname{grad} \phi_{l}\right\|_{\boldsymbol{L}^{2}\left(\Omega_{C}\right)} .
$$

Proof. Again, pick some $T \in \mathcal{T}_{m}^{C}$. Using the notation of the previous proof and $\operatorname{div} \mathbf{v}_{m \mid T}=0$, we infer from Green's formula that

$$
\begin{aligned}
\int_{T} \mathbf{v}_{m} \cdot \operatorname{grad} \phi_{l} d \mathbf{x} & =\int_{\partial T} \phi_{l} \mathbf{v}_{m} \cdot \mathbf{n} d S=\int_{\Sigma_{T}} \mathbf{v}_{m} \cdot \operatorname{grad} \phi_{l} d \mathbf{x} \\
& \lesssim h_{l} h_{m}^{-1}\left\|\mathbf{v}_{m}\right\|_{L^{2}(T)}\left\|\operatorname{grad} \phi_{l}\right\|_{\boldsymbol{L}^{2}(T)} .
\end{aligned}
$$

These two lemmas and the definition of the bilinear form $a(\cdot, \cdot)$ immediately yield the pivotal strengthened Cauchy-Schwarz inequality for the decomposition (4.1).

Theorem 4.4. For $0 \leq m \leq l \leq L$ and all admissible $i, j$ it holds that

$$
\begin{aligned}
\left|a\left(\mathbf{d}_{l}^{i}, \mathbf{d}_{m}^{j}\right)\right| & \lesssim h_{l} h_{m}^{-1} \min \left\{1, \sqrt{\frac{\beta_{0}}{\chi_{0}}}\right\}\left\|\mathbf{d}_{l}^{i}\right\|_{\mathfrak{E} ; \Omega}\left\|\mathbf{d}_{l}^{j}\right\|_{\mathfrak{E} ; \Omega} & \forall \mathbf{d}_{l}^{i} \in \mathcal{D}_{l}^{i}, \mathbf{d}_{m}^{j} \in \mathcal{D}_{m}^{j}, \\
\left|a\left(\mathbf{d}_{l}^{i}, \operatorname{grad} \phi_{m}^{j}\right)\right| & \lesssim h_{l} \sqrt{\frac{\beta_{0}}{\chi_{0}}}\left\|\mathbf{d}_{l}^{i}\right\|_{\mathfrak{E} ; \Omega}\left\|\operatorname{grad} \phi_{m}^{j}\right\|_{\mathfrak{E} ; \Omega} & \forall \mathbf{d}_{l}^{i} \in \mathcal{D}_{l}^{i}, \phi_{m}^{j} \in \mathcal{C}_{m}^{j}, \\
\left|a\left(\mathbf{d}_{m}^{i}, \operatorname{grad} \phi_{l}^{j}\right)\right| & \lesssim h_{l} h_{m}^{-1}\left\|\mathbf{d}_{m}^{i}\right\|_{\mathfrak{E} ; \Omega}\left\|\operatorname{grad} \phi_{m}^{l}\right\|_{\mathfrak{E} ; \Omega} & \forall \mathbf{d}_{m}^{i} \in \mathcal{D}_{m}^{i}, \phi_{l}^{j} \in \mathcal{C}_{l}^{j}, \\
\left|a\left(\operatorname{grad} \phi_{m}^{i}, \operatorname{grad} \phi_{l}^{j}\right)\right| & \lesssim h_{l} h_{m}^{-1}\left\|\operatorname{grad} \phi_{m}^{i}\right\|_{\mathfrak{E} ; \Omega}\left\|\operatorname{grad} \phi_{l}^{j}\right\|_{\mathfrak{E} ; \Omega} & \forall \phi_{m}^{i} \in \mathcal{C}_{m}^{i}, \phi_{l}^{j} \in \mathcal{C}_{l}^{j} .
\end{aligned}
$$




\section{Helmholtz-type DeCOMPosition}

In [36] the key idea was to consider the bilinear form of the variational problem (1.2) separately on the components of the $\boldsymbol{L}^{2}(\Omega)$-orthogonal Helmholtz-decomposition

$$
\boldsymbol{H}_{0}(\mathbf{c u r l} ; \Omega)=\operatorname{Ker}(\mathbf{c u r l}) \oplus \operatorname{Ker}(\mathbf{c u r l})^{\perp} .
$$

The reasoning was that the bilinear form when restricted to $\operatorname{Ker}(\mathbf{c u r l})^{\perp}$ behaved like an $H^{1}(\Omega)$-elliptic bilinear form and became amenable to standard nodal multilevel decompositions. Exploiting the continuous embedding $\boldsymbol{H}(\operatorname{div} ; \Omega) \cap \boldsymbol{H}_{0}(\boldsymbol{c u r l} ; \Omega) \subset$ $\boldsymbol{H}^{1}(\Omega)$ for convex or smooth domains (cf. [30, Prop. 3.1]), the analysis could largely stay in the customary framework for second order elliptic problems. The idea remains valid, but the plain Helmholtz-decomposition (5.1) may no longer be an adequate tool in a more general situation: firstly, in the presence of re-entrant corners, $\boldsymbol{H}(\operatorname{div} ; \Omega) \cap \boldsymbol{H}_{0}(\operatorname{curl} ; \Omega) \not \subset \boldsymbol{H}^{1}(\Omega)[26]$, and, secondly, a plain $\boldsymbol{L}^{2}(\Omega)$ orthogonal splitting ignores the distinction between $\Omega_{C}$ and $\Omega_{e}$. We conclude that more elaborate splittings into the kernel of curl and some, by no means orthogonal, complements are needed.

Let us start with topological preliminaries. If $\Omega_{C}$ has $p \in \mathbb{N}_{0}$ holes, i.e., the first Betti number of $\Omega_{C}$ equals $p$, then we can find $p$ orientable cutting (Seifert-)surfaces $\Sigma_{1}, \ldots, \Sigma_{p}$ such that $\Omega_{C} \backslash\left(\Sigma_{1} \cup \cdots \cup \Sigma_{p}\right)$ admits a single-valued scalar potential for any irrotational vector field and $\partial \Sigma_{k} \subset \partial \Omega_{C}$ for all $k \in\{1, \ldots, p\}$ (see [4] and [3, Sect. 3]). Even better, as $\Omega_{C}$ is triangulated by $\mathcal{T}_{0}^{C}$, these cutting surfaces can be chosen such that their closures are the union of closed faces of elements of $\mathcal{T}_{0}^{C}$. This is what we take for granted from now on.

Write $\nu_{k} \in H^{1}\left(\Omega_{C} \backslash \Sigma_{k}\right) / \mathbb{R}, k=1, \ldots, p$, for the solutions of the transmission problems

$$
\Delta \nu_{k}=0 \text { in } \Omega_{C} \backslash \Sigma_{k}, \quad \frac{\partial \nu_{k}}{\partial \mathbf{n}}=0 \text { on } \partial \Omega_{C}, \quad\left[\nu_{k}\right]_{\Sigma_{k}}=1, \quad\left[\frac{\partial \nu_{k}}{\partial \mathbf{n}}\right]_{\Sigma_{k}}=0,
$$

where $[\cdot]_{\Sigma}$ stands for the jump of a function across $\Sigma$. Their gradients provide a complete set of harmonic Neumann vector fields for $\Omega_{C}$ [3, Prop. 3.14]. We introduce certain discrete counterparts $\mathbf{h}_{k}^{N} \in \mathcal{N}_{\mathcal{D}_{1}^{0}}\left(\Omega_{C} ; \mathcal{T}_{0}^{C}\right)$ defined by $\mathbf{h}_{k}^{N}:=$ $\widetilde{\operatorname{grad}} Q_{0}^{C} \nu_{k}, k=1, \ldots, p$, where $Q_{0}^{C}: H^{1}\left(\Omega_{C} \backslash \Sigma_{k}\right) \mapsto \mathcal{S}_{1}\left(\Omega_{C} \backslash \Sigma_{k} ; \mathcal{T}_{0}\right)$ is a ScottZhang type quasi-interpolation operator and grad is the gradient of a function in $H^{1}\left(\Omega_{C} \backslash \Sigma_{k}\right)$ regarded as a vector field in $\boldsymbol{L}^{2}\left(\Omega_{C}\right)$.

Lemma 5.1. All $\mathbf{u} \in \boldsymbol{H}^{0}\left(\mathbf{c u r l} ; \Omega_{C}\right)$ have a representation $\mathbf{u}=\operatorname{grad} \phi+\sum_{k} \alpha_{k} \mathbf{h}_{k}^{N}$, $\phi \in H^{1}\left(\Omega_{C}\right) / \mathbb{R}, \alpha_{k} \in \mathbb{R}, k=1, \ldots, p$, which fulfills

$$
\|\phi\|_{H^{1}\left(\Omega_{C}\right)}+\sum_{k=1}^{p}\left\|\alpha_{k} \mathbf{h}_{k}^{N}\right\|_{L^{2}\left(\Omega_{C}\right)} \lesssim\|\mathbf{u}\|_{L^{2}\left(\Omega_{C}\right)} .
$$

Proof. Theorem 3.12 of [3] implies that there is an $\boldsymbol{L}^{2}\left(\Omega_{C}\right)$-orthogonal representation

$$
\mathbf{u}=\operatorname{grad} \phi+\sum_{k=1}^{p} \widetilde{\widetilde{\operatorname{grad}}} \nu_{k}, \quad \phi \in H^{1}\left(\Omega_{C}\right), \alpha_{k} \in \mathbb{R} .
$$

The constant jump of $\nu_{k}$ across $\Sigma_{k}$ is inherited by $Q_{0}^{C} \nu_{k}$ owing to the special properties of the quasi-interpolation operator. Hence, $\nu_{k}-Q_{0}^{C} \nu_{k} \in H^{1}\left(\Omega_{C}\right)$. In 
addition we have the stability

$$
\left\|\widetilde{\operatorname{grad}} Q_{0}^{C} \nu_{k}\right\|_{L^{2}\left(\Omega_{C}\right)} \lesssim\left\|\widetilde{\operatorname{grad}} \nu_{k}\right\|_{L^{2}\left(\Omega_{C}\right)} .
$$

The assertion of the lemma is straightforward.

Next, we introduce the harmonic Dirichlet vector fields in $\Omega_{e}$. Denote by $\Gamma_{1}, \ldots, \Gamma_{q}, q \in \mathbb{N}$, the connected components of $\partial \Omega_{C}$ and let $\delta_{k} \in H^{1}\left(\Omega_{e}\right)$, $k=1, \ldots, q$, be the solutions of the Dirichlet problems

$$
\Delta \delta=0 \text { in } \Omega_{e}, \quad \delta_{\mid \partial \Omega}=0, \quad \delta_{\mid \Gamma_{i}}=0 \text { for } i \neq k, \quad \delta_{\mid \Gamma_{k}}=1 .
$$

Then the space of harmonic Dirichlet vector fields in $\Omega_{e}$ is given by

$$
\mathcal{H}^{D}\left(\Omega_{e}\right):=\operatorname{Span}\left\{\operatorname{grad} \delta_{k}, k=1 \ldots, q\right\} \subset \boldsymbol{H}_{0}^{0}\left(\operatorname{curl} ; \Omega_{e}\right) \cap \boldsymbol{H}^{0}\left(\operatorname{div} ; \Omega_{e}\right)
$$

(see [3 Prop. 3.18]). Discrete counterparts $\mathbf{h}_{k}^{D}$ are defined as above by $\mathbf{h}_{k}^{D}:=$ $\operatorname{grad} Q_{0}^{e} \delta_{k}$, where $Q_{0}^{e}: H^{1}\left(\Omega_{e}\right) \mapsto \mathcal{S}_{1}\left(\Omega_{e} ; \mathcal{T}_{0}^{e}\right)$ is another quasi-interpolation operator. Please be aware that $\mathbf{h}_{k}^{D} \in \mathcal{N}^{0}{ }_{1,0}^{0}\left(\Omega_{e} ; \mathcal{T}_{0}^{e}\right)$. An analogous result holds, the proof being almost the same as before:

Lemma 5.2. For $\mathbf{u} \in \boldsymbol{H}_{0}^{0}\left(\operatorname{curl} ; \Omega_{e}\right)$, there exists $\phi \in H_{0}^{1}\left(\Omega_{e}\right)$ and $\alpha_{k} \in \mathbb{R}, k=$ $1, \ldots, q$, such that $\mathbf{u}=\operatorname{grad} \phi+\sum_{k} \alpha_{k} \mathbf{h}_{k}^{D}$ and

$$
\|\phi\|_{H^{1}\left(\Omega_{e}\right)}+\sum_{k=1}^{q}\left\|\alpha_{k} \mathbf{h}_{k}^{D}\right\|_{L^{2}\left(\Omega_{e}\right)} \lesssim\|\mathbf{u}\|_{L^{2}\left(\Omega_{e}\right)} .
$$

The next theorem supplies the crucial Helmholtz-type decomposition.

Theorem 5.3. Under the assumptions on $\Omega$ and $\Omega_{C}$ made in Section 1 and given a coarse grid $\mathcal{T}_{0}$ as introduced in Section $\mathbf{3}$, we find for each $\mathbf{v} \in \boldsymbol{H}_{0}(\mathbf{c u r l} ; \Omega)$ a vector field

$$
\boldsymbol{\Psi} \in\left(\boldsymbol{H}^{1}\left(\Omega_{C}\right) \times \boldsymbol{H}^{1}\left(\Omega_{e}\right)\right) \cap \boldsymbol{H}_{0}(\operatorname{curl} ; \Omega),
$$

a function $\phi \in H_{0}^{1}(\Omega)$, and a finite element vector field $\mathbf{h}_{h} \in \mathcal{N}_{\mathcal{D}_{1,0}}\left(\Omega ; \mathcal{T}_{0}\right) \cap$ $\mathcal{N} \mathcal{D}_{1}^{0}\left(\Omega_{C} ; \mathcal{T}_{0}\right)$ such that

$$
\mathbf{v}=\mathbf{\Psi}+\operatorname{grad} \phi+\mathbf{h}_{h} .
$$

Moreover, we get the estimates

$$
\begin{aligned}
\|\boldsymbol{\Psi}\|_{H^{1}(\Omega)} & \lesssim\|\operatorname{curl} \mathbf{v}\|_{L^{2}(\Omega)}, \quad\|\phi\|_{H^{1}\left(\Omega_{C}\right)} \lesssim\|\mathbf{v}\|_{\boldsymbol{H}\left(\operatorname{curl} ; \Omega_{C}\right)}, \\
\left\|\mathbf{h}_{h}\right\|_{\boldsymbol{H}\left(\operatorname{curl} ; \Omega_{C}\right)} & \lesssim\|\mathbf{v}\|_{\boldsymbol{H}\left(\mathbf{c u r l} ; \Omega_{C}\right)}, \quad\left\|\operatorname{curl} \mathbf{h}_{h}\right\|_{L^{2}\left(\Omega_{e}\right)} \lesssim\|\mathbf{v}\|_{\boldsymbol{H}\left(\mathbf{c u r l} ; \Omega_{C}\right)} .
\end{aligned}
$$

Proof. Following the proof of Thm. 3.1 in [30, Sect.3.1], set $\mathbf{u}:=\operatorname{curl} \mathbf{v}_{\mid \Omega_{C}} \in$ $\boldsymbol{H}^{0}\left(\operatorname{div} ; \Omega_{C}\right)$ and define $\omega \in H^{1}\left(\Omega_{e}\right) / \mathbb{R}$ through

$$
\Delta \omega=0 \quad \text { in } \Omega_{e}, \quad \frac{\partial \omega}{\partial \mathbf{n}}=\mathbf{u} \cdot \mathbf{n} \quad \text { on } \partial \Omega_{C}, \quad \frac{\partial \omega}{\partial \mathbf{n}}=0 \quad \text { on } \partial \Omega .
$$

This permits us to define an extension $\tilde{\mathbf{u}} \in \boldsymbol{H}^{0}\left(\operatorname{div} ; \mathbb{R}^{3}\right)$ of $\mathbf{u}$ by

$$
\tilde{\mathbf{u}}(\mathbf{x})= \begin{cases}\mathbf{u}(\mathbf{x}), & \text { if } \mathbf{x} \in \Omega_{C}, \\ \operatorname{grad} \omega(\mathbf{x}), & \text { if } \mathbf{x} \in \Omega_{e}, \\ 0, & \text { if } \mathbf{x} \notin \Omega\end{cases}
$$


By Fourier transform (cf. proof of Lemma 3.5 in [3]) we find $\tilde{\boldsymbol{\Psi}} \in H^{1}\left(\mathbb{R}^{3}\right)$ such that $\operatorname{curl} \tilde{\boldsymbol{\Psi}}=\tilde{\mathbf{u}}, \operatorname{div} \tilde{\boldsymbol{\Psi}}=0$, and (cf. Thm. 2.5 of 30 , Sect. 2.2])

$$
\begin{aligned}
\|\tilde{\boldsymbol{\Psi}}\|_{\boldsymbol{H}^{1}\left(\mathbb{R}^{3}\right)} & \lesssim\|\tilde{\mathbf{u}}\|_{\boldsymbol{L}^{2}\left(\mathbb{R}^{3}\right)} \lesssim\|\mathbf{u} \cdot \mathbf{n}\|_{\boldsymbol{H}^{-\frac{1}{2}}\left(\partial \Omega_{C}\right)}+\|\mathbf{u}\|_{\boldsymbol{H}\left(\mathrm{div} ; \Omega_{C}\right)} \\
& \lesssim\|\mathbf{u}\|_{\boldsymbol{L}^{2}\left(\Omega_{C}\right)} \lesssim\|\mathbf{c u r l} \mathbf{v}\|_{\boldsymbol{L}^{2}\left(\Omega_{C}\right)} .
\end{aligned}
$$

We denote by $\boldsymbol{\Psi}_{C}$ its restriction to $\Omega_{C}$. Next, set $\mathbf{q}:=\mathbf{v}_{\mid \Omega_{C}}-\boldsymbol{\Psi}_{C}$, which means that curlq $=0$ in $\Omega_{C}$. According to Lemma 5.1 this implies the existence of $\phi_{C} \in H^{1}\left(\Omega_{C}\right) / \mathbb{R}$ and $\mathbf{h}_{h}^{C} \in \mathcal{N} \mathcal{D}_{1}^{0}\left(\Omega_{C} ; \mathcal{T}_{0}^{C}\right)$ such that $\mathbf{q}=\operatorname{grad} \phi_{C}+\mathbf{h}_{h}^{C}$ and

$$
\left\|\phi_{C}\right\|_{H^{1}\left(\Omega_{C}\right)}+\left\|\mathbf{h}_{h}^{C}\right\|_{L^{2}\left(\Omega_{C}\right)} \lesssim\|\mathbf{q}\|_{L^{2}\left(\Omega_{C}\right)} .
$$

As a consequence

$$
\left\|\operatorname{grad} \phi_{C}\right\|_{L^{2}\left(\Omega_{C}\right)} \lesssim\left(\|\Psi\|_{L^{2}\left(\Omega_{C}\right)}+\|\mathbf{v}\|_{L^{2}\left(\Omega_{C}\right)}\right) \lesssim\|\mathbf{v}\|_{H\left(\text { curl } ; \Omega_{C}\right)} .
$$

Combining the above inequalities, we have arrived at a decomposition

$$
\mathbf{v}_{\mid \Omega_{C}}=\boldsymbol{\Psi}_{C}+\operatorname{grad} \phi_{C}+\mathbf{h}_{h}^{C}, \quad \boldsymbol{\Psi}_{C} \in \boldsymbol{H}^{1}\left(\Omega_{C}\right), \phi_{C} \in H^{1}\left(\Omega_{C}\right),
$$

which satisfies $\left\|\mathbf{h}_{h}^{C}\right\|_{\boldsymbol{L}^{2}\left(\Omega_{C}\right)} \lesssim\|\mathbf{v}\|_{\boldsymbol{H}\left(\mathbf{c u r l} ; \Omega_{C}\right)}$ and

$$
\left\|\boldsymbol{\Psi}_{C}\right\|_{\boldsymbol{H}^{1}\left(\Omega_{C}\right)} \lesssim\|\operatorname{curl} \mathbf{v}\|_{L^{2}\left(\Omega_{C}\right)}, \quad\left\|\operatorname{grad} \phi_{C}\right\|_{L^{2}\left(\Omega_{C}\right)} \lesssim\|\mathbf{v}\|_{\boldsymbol{H}\left(\mathbf{c u r l} ; \Omega_{C}\right)} .
$$

The next step in the proof centers around extensions. Let $\tilde{\phi}_{C} \in H_{0}^{1}(\Omega)$ denote the harmonic extension of $\phi_{C}$. We also employ an $\boldsymbol{H}^{1}(\Omega)$-continuous extension of $\boldsymbol{\Psi}_{C}$ to $\tilde{\mathbf{\Psi}}_{C} \in \boldsymbol{H}_{0}^{1}(\Omega)$. Eventually, we perform a discrete extension of $\mathbf{h}_{h}^{C}$ to $\tilde{\mathbf{h}}_{h} \in \mathcal{N}_{\mathcal{D}_{1}}\left(\Omega ; \mathcal{T}_{0}\right)$. This can be done by simply setting d.o.f. associated with edges in $\Omega_{e}$ to zero. Even this crude procedure results in

$$
\left\|\tilde{\mathbf{h}}_{h}\right\|_{\boldsymbol{H}(\operatorname{curl} ; \Omega)} \lesssim\left\|\mathbf{h}_{h}^{C}\right\|_{L^{2}\left(\Omega_{C}\right)} .
$$

Set

$$
\mathbf{z}:=\mathbf{v} \mid \Omega_{e}-\tilde{\boldsymbol{\Psi}}_{C}-\operatorname{grad} \tilde{\phi_{C}}-\tilde{\mathbf{h}}_{h} \in \boldsymbol{H}_{0}\left(\operatorname{curl} ; \Omega_{e}\right)
$$

and observe

$$
\|\mathbf{z}\|_{\boldsymbol{H}\left(\operatorname{curl} ; \Omega_{e}\right)} \lesssim\|\mathbf{v}\|_{\boldsymbol{H}\left(\operatorname{curl} ; \Omega_{e}\right)}+\left\|\Psi_{C}\right\|_{H^{1}\left(\Omega_{C}\right)}+\left\|\operatorname{grad} \phi_{C}\right\|_{\boldsymbol{L}^{2}\left(\Omega_{C}\right)}+\left\|\mathbf{h}_{h}^{C}\right\|_{\boldsymbol{L}^{2}\left(\Omega_{C}\right)}
$$

which means $\|\mathbf{z}\|_{\boldsymbol{H}\left(\mathbf{c u r l} ; \Omega_{e}\right)} \lesssim\|\mathbf{v}\|_{\boldsymbol{H}(\mathbf{c u r l} ; \Omega)}$. Consider the $\boldsymbol{L}^{2}\left(\Omega_{e}\right)$-orthogonal Helmholtz decomposition

$$
\mathbf{z}=\mathbf{\Theta}^{\prime}+\operatorname{grad} \nu^{\prime}, \quad \Theta^{\prime} \in \mathbf{X}_{N}\left(\Omega_{e}\right):=\boldsymbol{H}_{0}\left(\operatorname{curl} ; \Omega_{e}\right) \cap \boldsymbol{H}\left(\operatorname{div} ; \Omega_{e}\right), \nu^{\prime} \in H_{0}^{1}\left(\Omega_{e}\right) .
$$

Next, use Thm. 3.1 from [9] (see also [10, Prop. 5.1] and [27] Thm. 3.4]), which states the existence of a stable splitting

$$
\boldsymbol{\Theta}^{\prime}=\boldsymbol{\Theta}+\operatorname{grad} \nu, \quad \boldsymbol{\Theta} \in \boldsymbol{H}^{1}\left(\Omega_{e}\right) \cap \boldsymbol{H}_{0}\left(\operatorname{curl} ; \Omega_{e}\right), \quad \nu \in H_{0}^{1}\left(\Omega_{e}\right) .
$$

Since $\operatorname{div} \boldsymbol{\Theta}^{\prime}=0$, stability of the splitting means that

$$
\|\Theta\|_{H^{1}\left(\Omega_{e}\right)} \lesssim\left(\left\|\Theta^{\prime}\right\|_{L^{2}\left(\Omega_{e}\right)}+\left\|\operatorname{curl} \Theta^{\prime}\right\|_{L^{2}\left(\Omega_{e}\right)}\right) \lesssim\|\operatorname{curl} \mathbf{z}\|_{L^{2}\left(\Omega_{e}\right)}
$$

On top of that, $\operatorname{curl} \boldsymbol{\Theta}=\operatorname{curl} \Theta^{\prime}$, so that $\mathbf{z}-\boldsymbol{\Theta} \in \boldsymbol{H}_{0}^{0}\left(\operatorname{curl} ; \Omega_{e}\right)$. By Lemma 5.2 there exist $\phi_{e} \in H_{0}^{1}\left(\Omega_{e}\right) / \mathbb{R}$ and $\mathbf{h}_{h}^{e} \in \mathcal{N} \mathcal{D}_{1,0}^{0}\left(\Omega_{e} ; \mathcal{T}_{0}^{e}\right)$ such that $\mathbf{z}-\boldsymbol{\Theta}=\operatorname{grad} \phi_{e}+\mathbf{h}_{h}^{e}$ and

$$
\left\|\phi_{e}\right\|_{H^{1}\left(\Omega_{e}\right)}+\left\|\mathbf{h}_{h}^{e}\right\|_{L^{2}\left(\Omega_{e}\right)} \lesssim\|\mathbf{z}-\Theta\|_{L^{2}\left(\Omega_{e}\right)}
$$


Finally we end up with the decomposition

$$
\mathbf{v}= \begin{cases}\mathbf{\Psi}_{C}+\operatorname{grad} \phi_{C}+\tilde{\mathbf{h}}_{h}^{C}, & \text { in } \Omega_{C}, \\ \left(\tilde{\mathbf{\Psi}}_{C}+\boldsymbol{\Theta}\right)+\operatorname{grad}\left(\phi_{e}+\tilde{\phi}_{C}\right)+\left(\tilde{\mathbf{h}}_{h}+\mathbf{h}_{h}^{e}\right), & \text { in } \Omega_{e} .\end{cases}
$$

This, written as $\mathbf{v}=\mathbf{\Psi}+\operatorname{grad} \phi+\mathbf{h}_{h}$, is the asserted splitting.

\section{Stability estimate}

The verification of the stability (3.6) of the multilevel decomposition (3.1) boils down to finding a concrete stable splitting compatible with (3.1) for an arbitrary $\mathbf{u}_{h} \in \mathcal{N} \mathcal{D}_{1,0}\left(\Omega ; \mathcal{T}_{L}\right)$. By Theorem 5.3 we may write

$$
\mathbf{u}_{h}=\mathbf{\Psi}+\operatorname{grad} \phi+\mathbf{h}_{h},
$$

where the individual components belong to the spaces specified in the statement of Theorem 5.3 Keep in mind that both $\boldsymbol{\Psi}$ and $\phi$ have no reason to belong to any finite element space. We have to treat them as rather general functions, which accounts for most of the difficulties we are facing.

The construction of a particular decomposition of $\mathbf{u}_{h}$ is carried out separately for the terms in (6.1) and proceeds in several steps. A crude road map is as follows:

(1) Find stable nodal multilevel decompositions of $\boldsymbol{\Psi}$ minus some gradient separately in $\Omega_{C}$ and $\Omega_{e}$. The components belong to the $\widehat{\mathcal{N D}}_{1}$-spaces.

(2) Alter the decomposition in $\Omega_{e}$ such that it meets boundary and matching conditions on $\partial \Omega$ and $\Gamma$, respectively.

(3) Return to the lowest order edge element spaces by shedding suitable gradients.

(4) Provide a stable multilevel decomposition for the accumulated gradients of (6.1) and the previous stages.

The first item is settled by the next lemma.

Lemma 6.1. We can find $\boldsymbol{\Phi}_{l}^{C} \in \widehat{\mathcal{N D}}_{1}\left(\Omega_{C} ; \mathcal{T}_{l}^{C}\right), \boldsymbol{\Phi}_{l}^{e} \in \widehat{\mathcal{N D}}_{1}\left(\Omega_{e} ; \mathcal{T}_{l}^{e}\right), l=0, \ldots, L$, and $\sigma^{C} \in H^{1}(\Omega), \sigma^{e} \in H^{1}\left(\Omega_{e}\right)$ such that for $\boldsymbol{\Psi}$ from (6.1)

$$
\boldsymbol{\Psi}=\left\{\begin{array}{cc}
\sum_{l=0}^{L} \boldsymbol{\Phi}_{l}^{C}-\operatorname{grad} \sigma^{C} & \text { in } \Omega_{C}, \\
\sum_{l=0}^{L} \boldsymbol{\Phi}_{l}^{e}-\operatorname{grad} \sigma^{e} & \text { in } \Omega_{e},
\end{array}\right.
$$

and

$$
\begin{gathered}
\left\|\boldsymbol{\Phi}_{0}^{C}\right\|_{\boldsymbol{H}\left(\mathbf{c u r l} ; \Omega_{C}\right)}^{2}+\sum_{l=1}^{L} h_{l}^{-2}\left\|\boldsymbol{\Phi}_{l}^{C}\right\|_{L^{2}\left(\Omega_{C}\right)}^{2} \lesssim|\boldsymbol{\Psi}|_{H^{1}\left(\Omega_{C}\right)}^{2}, \\
\left\|\boldsymbol{\Phi}_{0}^{e}\right\|_{\boldsymbol{H}\left(\mathbf{c u r l} ; \Omega_{e}\right)}^{2}+\sum_{l=1}^{L} h_{l}^{-2}\left\|\boldsymbol{\Phi}_{l}^{e}\right\|_{\boldsymbol{L}^{2}\left(\Omega_{e}\right)}^{2} \lesssim\|\boldsymbol{\Psi}\|_{H^{1}\left(\Omega_{e}\right)}^{2}, \\
\left\|\sigma^{C}\right\|_{H^{1}\left(\Omega_{C}\right)} \lesssim h_{L}\|\boldsymbol{\Psi}\|_{H^{1}\left(\Omega_{C}\right)}, \quad\left\|\sigma^{e}\right\|_{H^{1}\left(\Omega_{e}\right)} \lesssim h_{L}\|\boldsymbol{\Psi}\|_{H^{1}\left(\Omega_{e}\right)} .
\end{gathered}
$$

Proof. First, we use a fundamental result about the nodal multilevel decomposition of $\mathcal{S}_{1}\left(\Omega ; \mathcal{T}_{L}\right)$ underlying the BPX-preconditioner (cf. [50], appendix of [59], and [64]). 
We apply it to the Cartesian components of $\mathbf{Q}_{L}^{C} \mathbf{\Psi}$, where $\mathbf{Q}_{L}^{C}: \boldsymbol{H}^{1}(\Omega) \mapsto \mathcal{S}_{1}\left(\Omega ; \mathcal{T}_{L}^{C}\right)$ is a vector-valued quasi-interpolation operator. We get a multilevel decomposition

$$
\mathbf{Q}_{L}^{C} \boldsymbol{\Psi}=\sum_{l=0}^{L} \widetilde{\boldsymbol{\Phi}}_{l}^{C}, \quad \widetilde{\boldsymbol{\Phi}}_{l}^{C} \in \mathcal{S}_{1}\left(\Omega ; \mathcal{T}_{l}^{C}\right)
$$

which fulfills

$$
\left|\widetilde{\boldsymbol{\Phi}}_{0}^{C}\right|_{H^{1}\left(\Omega_{C}\right)}^{2}+\sum_{l=1}^{L} h_{l}^{-2}\left\|\widetilde{\boldsymbol{\Phi}}_{l}^{C}\right\|_{\boldsymbol{L}^{2}\left(\Omega_{C}\right)}^{2} \lesssim\left|\mathbf{Q}_{L}^{C} \boldsymbol{\Psi}\right|_{H^{1}\left(\Omega_{C}\right)}^{2} \lesssim|\boldsymbol{\Psi}|_{H^{1}\left(\Omega_{C}\right)}^{2} .
$$

The idea is to incorporate the difference $\boldsymbol{\Psi}-\mathbf{Q}_{L}^{C} \boldsymbol{\Psi}$ partly into $\widetilde{\boldsymbol{\Phi}}_{L}^{C}$ and partly into a gradient. Set

$$
\boldsymbol{\Theta}_{l}^{C}:= \begin{cases}\widetilde{\boldsymbol{\Phi}}_{l}^{C}, & \text { for } 0 \leq l<L, \\ \widetilde{\boldsymbol{\Phi}}_{L}^{C}+\left(\mathbf{\Psi}-\mathbf{Q}_{L}^{C} \mathbf{\Psi}\right), & \text { for } l=L .\end{cases}
$$

Then

$$
\sum_{l=0}^{L} \boldsymbol{\Theta}_{l}^{C}=\boldsymbol{\Psi}, \quad \boldsymbol{\Theta}_{l}^{C} \in \mathcal{S}_{1}\left(\Omega ; \mathcal{T}_{l}\right) \text { for } 0 \leq l<L, \text { but } \boldsymbol{\Theta}_{L}^{C} \in \boldsymbol{H}^{1}(\Omega),
$$

and thanks to the approximation estimate

$$
\left\|\Psi-\mathbf{Q}_{L}^{C} \boldsymbol{\Psi}\right\|_{L^{2}(\Omega)} \lesssim h_{L}|\Psi|_{H^{1}(\Omega)},
$$

the asymptotic stability of the new splitting is maintained:

$$
\left|\boldsymbol{\Theta}_{0}^{C}\right|_{H^{1}\left(\Omega_{C}\right)}^{2}+\sum_{l=1}^{L} h_{l}^{-2}\left\|\Theta_{l}^{C}\right\|_{L^{2}\left(\Omega_{C}\right)}^{2} \lesssim|\Psi|_{H^{1}\left(\Omega_{C}\right)}^{2} .
$$

However, $\boldsymbol{\Theta}_{L}^{C}$ is no longer a discrete vector field. Yet, its curl is a finite element function in $\mathcal{R} \mathcal{T}_{0}\left(\Omega_{C} ; \mathcal{T}_{L}\right)$, because

$$
\operatorname{curl} \Theta_{L}^{C}=\operatorname{curl}\left(\mathbf{u}_{h}-\mathbf{h}_{h}\right)-\operatorname{curl}\left(\sum_{l=0}^{L-1} \Theta_{l}^{C}\right) .
$$

This carries the important consequence that the nodal interpolation operator $\widehat{\Pi}_{h}$ onto $\widehat{\mathcal{N D}}_{1}\left(\Omega ; \mathcal{T}_{L}^{C}\right)$ is well defined for $\boldsymbol{\Theta}_{L}^{C}$ and that the interpolation error can be estimated. As can be seen from both [40, Lemma 4.3] and [23, Lemma 3.3], the interpolation estimate

$$
\left\|\Theta_{L}^{C}-\widehat{\Pi}_{h} \Theta_{L}^{C}\right\|_{L^{2}\left(\Omega_{C}\right)} \lesssim h_{L}\left|\Theta_{L}^{C}\right|_{H^{1}\left(\Omega_{C}\right)} \lesssim h_{L}\|\Psi\|_{H^{1}(\Omega)}
$$

holds true. This enables us to set

$$
\boldsymbol{\Phi}_{L}^{C}:=\widehat{\Pi}_{h} \Theta_{L \mid \Omega_{C}}^{C} \in \widehat{\mathcal{N D}}_{1}\left(\Omega ; \mathcal{T}_{L}^{C}\right) .
$$

By the commuting diagram property (2.5) we conclude that

$$
\operatorname{curl}\left(\Theta_{L}^{C}-\boldsymbol{\Phi}_{L}^{C}\right)=0 \quad \text { in } \Omega_{C}
$$

Now apply Lemma 5.1 to $\boldsymbol{\Phi}_{L}^{C}-\boldsymbol{\Theta}_{L}^{C}$, which yields a representation

$$
\boldsymbol{\Phi}_{L}^{C}-\boldsymbol{\Theta}_{L}^{C}=\operatorname{grad} \sigma^{C}+\mathbf{w}_{h}, \quad \sigma^{C} \in H^{1}(\Omega), \mathbf{w}_{h} \in \widehat{\mathcal{N D}}_{1}^{0}\left(\Omega ; \mathcal{T}_{0}^{C}\right),
$$


and fix the remaining terms by

$$
\boldsymbol{\Phi}_{l}^{C}:= \begin{cases}\Theta_{l}^{C}, & \text { for } 0<l<L, \\ \Theta_{0}^{C}+\mathbf{w}_{h}, & \text { for } l=0 .\end{cases}
$$

The estimates of the lemma follow from those in Lemma 5.1 (6.2) and (6.3). The assertion for $\Omega_{e}$ can be shown in an entirely analogous fashion.

We have found multilevel decompositions of $\boldsymbol{\Psi}$, but their components neither match across $\partial \Omega_{C}$, i.e., they do not display any tangential continuity there, nor do the $\boldsymbol{\Phi}^{e}$ satisfy the boundary conditions at $\partial \Omega$. Thus, in a next step we have to alter the splittings to enforce matching at $\partial \Omega_{C}$ and $\partial \Omega$. At present, we know

$$
\begin{aligned}
& \mathbf{u}_{h}=\sum_{l=0}^{L} \boldsymbol{\Phi}_{l}^{C}+\operatorname{grad} \sigma^{C}+\operatorname{grad} \phi+\mathbf{h}_{h} \quad \text { in } \Omega_{C}, \\
& \mathbf{u}_{h}=\sum_{l=0}^{L} \boldsymbol{\Phi}_{l}^{e}+\operatorname{grad} \sigma^{e}+\operatorname{grad} \phi+\mathbf{h}_{h} \quad \text { in } \Omega_{e} .
\end{aligned}
$$

We get $\tilde{\sigma}^{C} \in H_{0}^{1}(\Omega)$ by extending $\sigma^{C}$ and write $\tau_{h}:=\widehat{Q}_{L}\left(\sigma^{e}-\tilde{\sigma}^{C}\right)$. From (6.4) we learn that

$$
\left(\sigma^{e}-\tilde{\sigma}^{C}\right)_{\mid \partial \Omega_{C}} \in \mathcal{S}_{2}\left(\partial \Omega_{C} ; \mathcal{T}_{L \mid \partial \Omega_{C}}\right) \quad \text { and } \quad \sigma_{\mid \partial \Omega}^{e} \in \mathcal{S}_{2}\left(\partial \Omega ; \mathcal{T}_{L \mid \partial \Omega}\right) .
$$

As before, we infer

$$
\left(\sigma^{e}-\tilde{\sigma}^{C}\right)-\tau_{h} \in H_{0}^{1}\left(\Omega_{e}\right) .
$$

Since, from the stability of the quasi-interpolation operator and Lemma 6.1

$$
\left\|\operatorname{grad} \tau_{h}\right\|_{L^{2}\left(\Omega_{e}\right)} \leq\left\|\tilde{\sigma}^{C}\right\|_{H^{1}\left(\Omega_{e}\right)}+\left\|\sigma^{e}\right\|_{H^{1}\left(\Omega_{e}\right)} \lesssim h_{L}\|\Psi\|_{H^{1}(\Omega)},
$$

we can incorporate grad $\tau_{h}$ into $\boldsymbol{\Phi}_{L}^{e}$ without affecting the estimates of Lemma 6.1 This time we retain the notation $\boldsymbol{\Phi}_{L}^{e}$ for the modified component. Summing up, we have

$$
\mathbf{u}_{h}=\operatorname{grad} \phi^{\prime}+\mathbf{h}_{h}+\left\{\begin{array}{cc}
\sum_{l=0}^{L} \boldsymbol{\Phi}_{l}^{C} & \text { in } \Omega_{C}, \\
\sum_{l=0}^{L} \boldsymbol{\Phi}_{l}^{e} & \text { in } \Omega_{e},
\end{array}\right.
$$

where

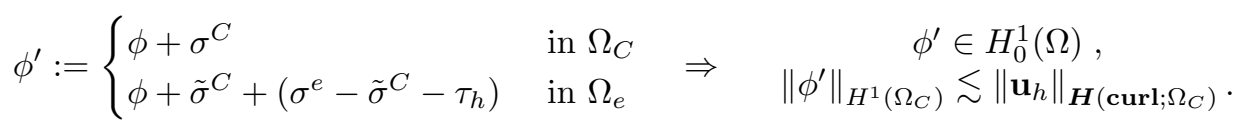

We have shown that

$$
\left(\sum_{l=0}^{L} \boldsymbol{\Phi}_{l}^{C}-\sum_{l=0}^{L} \boldsymbol{\Phi}_{l}^{e}\right) \times \mathbf{n}_{\mid \partial \Omega_{C}}=0 \quad \text { and } \sum_{l=0}^{L} \boldsymbol{\Phi}_{l}^{e} \times \mathbf{n}_{\mid \partial \Omega}=0 .
$$

As in [36, Sect. 5.1], we can now apply Oswald's trick [49 Cor. 30] to make the individual components of the decompositions match. Starting with the decomposition (6.5), we set

$$
\tilde{\mathbf{s}}_{n}:=\sum_{l=0}^{n} \boldsymbol{\Phi}_{l}^{e}, \quad \mathbf{s}_{n}^{C}:=\sum_{l=0}^{n} \boldsymbol{\Phi}_{l}^{C}, \quad 0 \leq n \leq L .
$$


Then, $\mathbf{s}_{n} \in \widehat{\mathcal{N D}}_{1}\left(\Omega_{e} ; \mathcal{T}_{n}^{e}\right)$ emerges from $\tilde{\mathbf{s}}_{n}$, first by discarding all basis functions on the boundary $\partial \Omega$, and second by setting the degrees of freedom on $\partial \Omega_{C}$ to those of $\mathbf{s}_{n}^{C}$ :

$$
\mathbf{s}_{n}=\sum_{\kappa \in \hat{\Xi}^{\text {int }}\left(\mathcal{T}_{n}^{e}\right)} \kappa\left(\tilde{\mathbf{s}}_{n}\right) \hat{\mathbf{b}}_{\kappa}+\sum_{\kappa \in \widehat{\Xi}_{1}\left(\mathcal{T}_{n}^{\partial}\right)} \kappa\left(\mathbf{s}_{n}^{C}\right) \hat{\mathbf{b}}_{\kappa}
$$

where $\widehat{\Xi}^{\text {int }}\left(\mathcal{T}_{n}^{e}\right)$ designates the set of $\widehat{\mathcal{N D}}_{1}$-d.o.f. associated with edges in $\Omega_{e}$. Thanks to (6.6), Lemma 2.3 and (2.7) we can control the effect of the alterations:

$$
\begin{aligned}
\left\|\mathbf{s}_{n}-\tilde{\mathbf{s}}_{n}\right\|_{\boldsymbol{L}^{2}(\Omega)}^{2} \lesssim h_{n} \sum_{\kappa \in \hat{\Xi}_{1}\left(C t_{n}\right)} \kappa\left(\mathbf{s}_{n}-\tilde{\mathbf{s}}_{n}\right)^{2} \\
\vdots h_{n}\left(\left\|\tilde{\mathbf{s}}_{n} \times \mathbf{n}\right\|_{\boldsymbol{L}^{2}(\partial \Omega)}^{2}+\left\|\left(\mathbf{s}_{n}^{C}-\tilde{\mathbf{s}}_{n}\right) \times \mathbf{n}\right\|_{\boldsymbol{L}^{2}\left(\partial \Omega_{C}\right)}^{2}\right) \\
\vdots h_{n}\left(\left\|\left(\tilde{\boldsymbol{\Psi}}^{e}-\sum_{l=0}^{n} \boldsymbol{\Phi}_{l}^{e}\right) \times \mathbf{n}\right\|_{\boldsymbol{L}^{2}(\partial \Omega)}^{2}\right. \\
\left.\quad+\left\|\left(\tilde{\mathbf{\Psi}}^{e}-\sum_{l=0}^{n} \boldsymbol{\Phi}_{l}^{e}+\tilde{\mathbf{\Psi}}^{C}-\sum_{l=0}^{n} \boldsymbol{\Phi}_{l}^{C}\right) \times \mathbf{n}\right\|_{\boldsymbol{L}^{2}\left(\partial \Omega_{C}\right)}^{2}\right)
\end{aligned}
$$

where $\tilde{\boldsymbol{\Psi}}^{e}:=\sum_{l} \boldsymbol{\Phi}_{l}^{e}$ and $\tilde{\boldsymbol{\Psi}}^{C}:=\sum_{l} \boldsymbol{\Phi}_{l}^{C}$. The estimate of the first term will serve to elucidate the technique. First we make use of stability estimates (2.7) for the bases of $\mathcal{R} \mathcal{T}_{0}\left(\partial \Omega ; \mathcal{T}_{n}^{\partial}\right)$ and of Lemma 2.1 for $\widehat{\mathcal{N D}}_{1}\left(\Omega_{e} ; \mathcal{T}_{n}^{e}\right)$ :

$$
\left\|\left(\tilde{\boldsymbol{\Psi}}^{e}-\sum_{l=0}^{n} \boldsymbol{\Phi}_{l}^{e}\right) \times \mathbf{n}\right\|_{\boldsymbol{L}^{2}(\partial \Omega)} \leq \sum_{l=n+1}^{L}\left\|\boldsymbol{\Phi}_{l}^{e} \times \mathbf{n}\right\|_{\boldsymbol{L}^{2}(\partial \Omega)} \leq \sum_{l=n+1}^{L} h_{l}^{-1 / 2}\left\|\boldsymbol{\Phi}_{l}^{e}\right\|_{\boldsymbol{L}^{2}\left(\Omega_{e}\right)} .
$$

Thanks to $h_{l} \approx 2^{-l}$, we immediately get from the Cauchy-Schwarz inequality

$$
\sum_{l=n+1}^{L} h_{l}^{-1 / 2}\left\|\boldsymbol{\Phi}_{l}^{e}\right\|_{\boldsymbol{L}^{2}\left(\Omega_{e}\right)} \lesssim h_{n}^{1 / 2-\epsilon}\left(\sum_{l=n+1}^{L} h_{l}^{-2(1-\epsilon)}\left\|\boldsymbol{\Phi}_{l}^{e}\right\|_{\boldsymbol{L}^{2}\left(\Omega_{e}\right)}^{2}\right)^{1 / 2},
$$

for $0 \leq n \leq L$ and any small $\epsilon>0$. We continue by

$$
\begin{aligned}
& \sum_{l=1}^{L} h_{l}^{-1}\left\|\tilde{\mathbf{s}}_{l} \times \mathbf{n}\right\|_{L^{2}(\partial \Omega)}^{2} \lesssim \sum_{l=1}^{L} h_{l}^{-2 \epsilon} \sum_{i=l+1}^{L} h_{i}^{-2(1-\epsilon)}\left\|\boldsymbol{\Phi}_{i}^{e}\right\|_{L^{2}\left(\Omega_{e}\right)}^{2} \\
& \lesssim \sum_{i=2}^{L}\left(\sum_{l=1}^{i} h_{l}^{-2 \epsilon}\right) h_{i}^{-2(1-\epsilon)}\left\|\boldsymbol{\Phi}_{i}^{e}\right\|_{L^{2}\left(\Omega_{e}\right)}^{2} \lesssim \sum_{i=2}^{L} h_{i}^{-2}\left\|\boldsymbol{\Phi}_{i}^{e}\right\|_{\boldsymbol{L}^{2}\left(\Omega_{e}\right)}^{2} \lesssim\|\boldsymbol{\Psi}\|_{H^{1}\left(\Omega_{e}\right)}^{2}
\end{aligned}
$$

A similar treatment can be used to bound the other terms and yields

$$
\sum_{l=1}^{L} h_{l}^{-2}\left\|\mathbf{s}_{l}-\tilde{\mathbf{s}}_{l}\right\|_{\boldsymbol{L}^{2}(\Omega)}^{2} \lesssim\|\boldsymbol{\Psi}\|_{\boldsymbol{L}^{2}(\Omega)}^{2} .
$$

The partial sums $\mathbf{s}_{n}, n=0, \ldots, K$, now generate the desired components $\boldsymbol{\Phi}_{l} \in$ $\widehat{\mathcal{N D}}_{1}\left(\Omega_{e} ; \mathcal{T}_{l}\right), 0 \leq l \leq L$, satisfying the matching conditions by

$$
\mathbf{\Phi}_{l}:=\left\{\begin{array}{cl}
\mathbf{s}_{0} & \text { for } l=0, \\
\mathbf{s}_{l}-\mathbf{s}_{l-1} & \text { for } l \in\{1, \ldots, L\} .
\end{array}\right.
$$


Closer scrutiny reveals that for $l \geq 1$,

$$
\boldsymbol{\Phi}_{l}=\left(\mathbf{s}_{l}-\tilde{\mathbf{s}}_{l}\right)-\left(\mathbf{s}_{l-1}-\tilde{\mathbf{s}}_{l-1}\right)+\boldsymbol{\Phi}_{l}^{e} .
$$

Taking into account (6.8) and Theorem 5.3 we have confirmed the following lemma.

Lemma 6.2. For each $\mathbf{u}_{h} \in \mathcal{N}_{\mathcal{D}_{1,0}}\left(\Omega ; \mathcal{T}_{L}\right)$ we can find $\boldsymbol{\Phi}_{l} \in \widehat{\mathcal{N D}}_{1,0}\left(\Omega ; \mathcal{T}_{l}\right), 0 \leq$ $l \leq L, \mathbf{h}_{h} \in \mathcal{N}_{\mathcal{D}_{1,0}}\left(\Omega ; \mathcal{T}_{0}\right) \cap \mathcal{N D}_{1}^{0}\left(\Omega_{C} ; \mathcal{T}_{0}^{C}\right)$, and $\phi_{h} \in \mathcal{S}_{2,0}\left(\Omega ; \mathcal{T}_{L}\right)$ such that $\mathbf{u}_{h}=$ $\sum_{l=0}^{L} \boldsymbol{\Phi}_{l}+\operatorname{grad} \phi_{h}+\mathbf{h}_{h}$ and

$$
\begin{aligned}
\left\|\boldsymbol{\Phi}_{0}\right\|_{\boldsymbol{H}(\mathbf{c u r l} ; \Omega)}^{2}+\sum_{l=1}^{L} h_{l}^{-2}\left\|\boldsymbol{\Phi}_{l}\right\|_{\boldsymbol{L}^{2}(\Omega)}^{2} & \lesssim\left\|\operatorname{curl} \mathbf{u}_{h}\right\|_{\boldsymbol{L}^{2}(\Omega)}^{2}, \\
\left\|\phi_{h}\right\|_{H^{1}\left(\Omega_{C}\right)} & \lesssim\left\|\mathbf{u}_{h}\right\|_{\boldsymbol{H}\left(\mathbf{c u r l} ; \Omega_{C}\right)} .
\end{aligned}
$$

Proof. By and large, the preceding considerations have settled everything. I point out that $\operatorname{grad} \phi_{h} \in \widehat{\mathcal{N D}}_{1}\left(\Omega ; \mathcal{T}_{L}\right)$.

Still, we are not done, because we would like to have $\boldsymbol{\Phi}_{l} \in \mathcal{N} \mathcal{D}_{1,0}\left(\Omega ; \mathcal{T}_{l}\right)$ and, on top of that, we need to split the contribution $\phi_{h}$ of the potential space.

Theorem 6.3. For each $\mathbf{u}_{h} \in \mathcal{N} \mathcal{D}_{1,0}\left(\Omega ; \mathcal{T}_{L}\right)$ there exist $\mathbf{v}_{l} \in \mathcal{N} \mathcal{D}_{1,0}\left(\Omega ; \mathcal{T}_{l}\right), \mu_{l} \in$ $\mathcal{S}_{1,0}\left(\Omega ; \mathcal{T}_{l}\right), 0 \leq l \leq L$, and $\mathbf{h}_{h} \in \mathcal{N}_{\mathcal{D}_{1,0}}\left(\Omega ; \mathcal{T}_{0}\right) \cap \mathcal{N}_{\mathcal{D}_{1}^{0}}^{0}\left(\Omega_{C} ; \mathcal{T}_{0}^{C}\right)$ such that $\mathbf{u}_{h}=$ $\sum_{l=0}^{L} \mathbf{v}_{l}+\sum_{l=0}^{L} \operatorname{grad} \mu_{l}+\mathbf{h}_{h}$ and the following stability estimates are satisfied

$$
\begin{gathered}
\left\|\mathbf{v}_{0}\right\|_{\boldsymbol{H}(\operatorname{curl} ; \Omega)}^{2}+\sum_{l=1}^{L} h_{l}^{-2}\left\|\mathbf{v}_{l}\right\|_{\boldsymbol{L}^{2}(\Omega)}^{2} \lesssim\left\|\mathbf{c u r l} \mathbf{u}_{h}\right\|_{\boldsymbol{L}^{2}(\Omega)}^{2} \\
\left\|\mu_{0}\right\|_{H^{1}\left(\Omega_{C}\right)}^{2}+\sum_{l=1}^{L} h_{l}^{-2}\left\|\mu_{l}\right\|_{L^{2}\left(\Omega_{C}\right)}^{2} \lesssim\left\|\mathbf{u}_{h}\right\|_{\boldsymbol{H}\left(\operatorname{curl} ; \Omega_{C}\right)}^{2} \\
\left\|\mathbf{h}_{h}\right\|_{\boldsymbol{L}^{2}\left(\Omega_{C}\right)} \lesssim\left\|\mathbf{u}_{h}\right\|_{\boldsymbol{H}\left(\operatorname{curl} ; \Omega_{C}\right)}, \quad\left\|\operatorname{curl} \mathbf{h}_{h}\right\|_{L^{2}\left(\Omega_{e}\right)} \lesssim\left\|\mathbf{u}_{h}\right\|_{\boldsymbol{H}\left(\operatorname{curl} ; \Omega_{C}\right)} .
\end{gathered}
$$

Proof. We start with the decomposition $\mathbf{u}_{h}=\sum_{l=0}^{L} \boldsymbol{\Phi}_{l}+\operatorname{grad} \phi_{h}+\mathbf{h}_{h}$ from the previous lemma. According to Lemma 2.2 we can split $\boldsymbol{\Phi}_{l}=: \mathbf{v}_{l}+\operatorname{grad} \hat{\gamma}_{l}$, with $\mathbf{v}_{l} \in \mathcal{N} \mathcal{D}_{1,0}\left(\Omega ; \mathcal{T}_{l}\right)$ and $\hat{\gamma}_{l} \in \hat{\mathcal{S}}_{2,0}\left(\Omega ; \mathcal{T}_{l}\right)$ and

$$
\left\|\mathbf{v}_{l}\right\|_{\boldsymbol{L}^{2}(\Omega)}+\left\|\operatorname{grad} \hat{\gamma}_{l}\right\|_{\boldsymbol{L}^{2}(\Omega)} \lesssim\left\|\boldsymbol{\Phi}_{l}\right\|_{\boldsymbol{L}^{2}(\Omega)} .
$$

By the stability estimate from Lemma 6.2, one infers via the Cauchy-Schwarz inequality

$$
\begin{aligned}
\left\|\operatorname{grad} \sum_{l=0}^{L} \hat{\gamma}_{l}\right\|_{\boldsymbol{L}^{2}(\Omega)} & \leq \sum_{l=0}^{L}\left\|\operatorname{grad} \hat{\gamma}_{l}\right\|_{\boldsymbol{L}^{2}(\Omega)} \\
& \leq\left\|\operatorname{grad} \hat{\gamma}_{0}\right\|_{\boldsymbol{L}^{2}(\Omega)}+\sum_{l=1}^{L} h_{l}\left(h_{l}^{-1}\left\|\operatorname{grad} \hat{\gamma}_{l}\right\|_{\boldsymbol{L}^{2}(\Omega)}\right) \\
& \lesssim\left\|\boldsymbol{\Phi}_{0}\right\|_{\boldsymbol{L}^{2}(\Omega)}+\left(\sum_{l=1}^{L} h_{l}^{2}\right)^{\frac{1}{2}}\left(\sum_{l=1}^{L} h_{l}^{-2}\left\|\boldsymbol{\Phi}_{l}\right\|_{\boldsymbol{L}^{2}(\Omega)}^{2}\right)^{\frac{1}{2}} \\
& \lesssim\left\|\operatorname{curl} \mathbf{u}_{h}\right\|_{\boldsymbol{L}^{2}(\Omega)} .
\end{aligned}
$$


Everything remains valid, when replacing $\Omega$ by $\Omega_{C}$. Therefore, $\psi_{h}:=\phi_{h}+\sum_{l=0}^{L} \hat{\gamma}_{l}$ satisfies

$$
\left\|\psi_{h}\right\|_{H^{1}(\Omega)} \lesssim\left\|\mathbf{u}_{h}\right\|_{\boldsymbol{H}(\mathbf{c u r l} ; \Omega)} \quad \text { and } \quad\left\|\psi_{h}\right\|_{H^{1}\left(\Omega_{C}\right)} \lesssim\left\|\mathbf{u}_{h}\right\|_{\boldsymbol{H}\left(\mathbf{c u r l} ; \Omega_{C}\right)} .
$$

Applying standard results about stable BPX-type multilevel splittings for $H^{1}(\Omega)$ conforming quadratic Lagrangian finite elements gives $\mu_{l} \in \mathcal{S}_{1,0}\left(\Omega ; \mathcal{T}_{l}\right), 0 \leq l<L$, and $\mu_{L} \in \mathcal{S}_{2,0}\left(\Omega ; \mathcal{T}_{L}\right)$ such that

$$
\psi_{h}=\sum_{l=0}^{L} \mu_{l}, \quad\left\|\mu_{0}\right\|_{H^{1}\left(\Omega_{C}\right)}^{2}+\sum_{l=1}^{L} h_{l}^{-2}\left\|\mu_{l}\right\|_{L^{2}\left(\Omega_{C}\right)}^{2} \lesssim\left\|\psi_{h}\right\|_{H^{1}\left(\Omega_{C}\right)}^{2} .
$$

Due to

$$
\operatorname{grad} \mu_{L}=\mathbf{u}_{h}-\mathbf{h}_{h}-\sum_{l=0}^{L} \mathbf{v}_{l}-\sum_{l=0}^{L-1} \operatorname{grad} \mu_{l} \in \mathcal{N} \mathcal{D}_{1,0}^{0}\left(\Omega ; \mathcal{T}_{L}\right)
$$

$\mu_{L}$ has a piece-wise constant gradient, and belongs to $\mathcal{S}_{1,0}\left(\Omega ; \mathcal{T}_{L}\right)$.

Next, a standard argument using the $L^{2}$-stability of the bases $\left\{\mathbf{b}_{\kappa}\right\}_{\kappa \in \Xi_{1}\left(\mathcal{T}_{l}\right)}$, $l=1, \ldots, L,($ cf. Lemma 2.1) and (2.1) confirms

$$
\left\|\operatorname{curl}_{\mathbf{0}}\right\|_{L^{2}(\Omega)}^{2}+\sum_{l=1}^{L} \sum_{\kappa \in \Xi_{1}\left(\mathcal{T}_{l}\right)}\left\|\operatorname{curl} \mathbf{v}_{l, \kappa}\right\|_{L^{2}(\Omega)}^{2} \lesssim\left\|\operatorname{curl} \mathbf{u}_{h}\right\|_{L^{2}(\Omega)}^{2},
$$

where the notation of Theorem 6.3 have been used and

$$
\sum_{\kappa \in \Xi_{1}\left(\mathcal{T}_{l}\right)} \mathbf{v}_{l, \kappa}=\mathbf{v}_{l}, \quad \mathbf{v}_{l, \kappa} \in \operatorname{Span}\left\{\mathbf{b}_{\kappa}\right\}
$$

We also obtain

$$
\left\|\mathbf{v}_{0}\right\|_{L^{2}(\Omega)}^{2}+\sum_{l=1}^{L} \sum_{\kappa \in \Xi_{1}\left(\mathcal{T}_{l}\right)}\left\|\mathbf{v}_{l, \kappa}\right\|_{L^{2}(\Omega)}^{2} \lesssim\left\|\operatorname{curl} \mathbf{u}_{h}\right\|_{\boldsymbol{L}^{2}(\Omega)}^{2} .
$$

Along with the estimates for $\mathbf{h}_{h}$ from Theorem 5.3 this means that

$$
\left\|\mathbf{v}_{0}+\mathbf{h}_{h}\right\|_{\mathfrak{E} ; \Omega}^{2}+\sum_{l=1}^{L} \sum_{\kappa \in \Xi_{1}\left(\mathcal{T}_{l}\right)}\left\|\mathbf{v}_{l, \kappa}\right\|_{\mathfrak{E} ; \Omega}^{2} \lesssim\left(\chi_{0}+\beta_{0}\right)\left(\left\|\operatorname{curl} \mathbf{u}_{h}\right\|_{L^{2}(\Omega)}^{2}+\left\|\mathbf{u}_{h}\right\|_{L^{2}\left(\Omega_{C}\right)}^{2}\right) .
$$

For the irrotational components of the decomposition, we deduce from Theorem 6.3 that

$$
\left|\mu_{0}\right|_{H^{1}\left(\Omega_{C}\right)}^{2}+\sum_{l=1}^{L} \sum_{\mathbf{x} \in \mathcal{N}_{l}}\left|\mu_{l, \mathbf{x}}\right|_{H^{1}\left(\Omega_{C}\right)}^{2} \lesssim\left\|\operatorname{curl} \mathbf{u}_{h}\right\|_{L^{2}(\Omega)}^{2}+\left\|\mathbf{u}_{h}\right\|_{L^{2}\left(\Omega_{C}\right)}^{2}
$$

where

$$
\sum_{\mathbf{x} \in \mathcal{N}_{l}} \mu_{l, \mathbf{x}}=\mu_{l}, \quad \mu_{l, \mathbf{x}} \in \operatorname{Span}\left\{\psi_{\mathbf{x}}\right\} .
$$

Eventually, skipping some elementary manipulations, it turns out that for the decomposition (3.1) a possible bound for the stability constant from (3.6) is given by

$$
C_{\text {stab }}=1+\frac{\chi_{0}}{\beta_{0}}+\frac{\beta_{0}}{\chi_{0}} .
$$


Now we are in a position to apply the powerful results of variational multigrid theory. Using Thm. 4.1, Lemmas 4.5, 4.6, and Thm. 4.4 of [59] our Theorems 4.4 and 6.3 in conjunction with (6.9) lead to the main result.

Theorem 6.4. Using the notation introduced in Section [3, the spectral condition number of $\widetilde{B}_{L}^{\text {add }} \widetilde{A}_{L}$ satisfies

$$
\kappa\left(\widetilde{B}_{L}^{\text {add }} \widetilde{A}_{L}\right):=\frac{\lambda_{\max }\left(\widetilde{B}_{L}^{\text {add }} \widetilde{A}_{L}\right)}{\lambda_{\min }\left(\widetilde{B}_{L}^{\text {add }} \widetilde{A}_{L}\right)} \lesssim \sqrt{\frac{\beta_{0}}{\chi_{0}}}\left(\frac{\beta_{0}}{\chi_{0}}+\frac{\chi_{0}}{\beta_{0}}\right) .
$$

The convergence rate $\rho$ - measured in the energy seminorm $\|\cdot\|_{\mathfrak{E} ; \Omega}$ - of the multiplicative Schwarz method (multigrid) based on (3.1) will be bounded by

$$
\rho:=\left\|\widetilde{I d}-\widetilde{B}_{L}^{\mathrm{mul}} \widetilde{A}_{L}\right\|_{\mathfrak{E} ; \Omega} \leq 1-\frac{1}{C\left(1+\sqrt{\beta_{0} / \chi_{0}}\right)\left(1+\beta_{0} / \chi_{0}+\chi_{0} / \beta_{0}\right)},
$$

where $C>0$ only depends on $\Omega, \Omega_{C}$, and the shape regularity of the triangulations.

What does it mean that the statements of the previous theorem use the setting of factor spaces? For instance in the case of the multigrid method, it means that there is no information about the behavior of components of the iterates that lie in $\operatorname{Ker}(a)$. A more thorough discussion is given in [38, Sect. 3]. In the case of the additive Schwarz preconditioner, Theorem 6.4 implies that the method can be used in conjunction with the preconditioned conjugate gradient method. We know that the latter will then converge in the factor space with a rate only bounded by the spectral condition number $\kappa\left(\widetilde{B}_{L}^{\text {add }} \widetilde{A}_{L}\right)$ 41]. The convergence in $\operatorname{Ker}(a)$ cannot be controlled either.

Remark. The presence of the ratio $\beta_{0} / \chi_{0}$ in the the estimates of Theorem 6.4 is problematic, because this ratio might become very large in the context of implicit time stepping. If I had succeeded in finding a splitting similar to (6.1), but stable with respect to the $\boldsymbol{L}^{2}\left(\Omega_{C}\right)$-norm, then the dependence of $C_{\text {stab }}$ on $\beta_{0}$ and $\chi_{0}$ could be removed. The genuine Helmholtz decomposition is a specimen of an $\boldsymbol{L}^{2}\left(\Omega_{C}\right)$ stable splitting, but unfortunately it lacks the essential regularity properties, unless $\Omega_{C}$ is smooth or convex.

However, numerical evidence suggests that for additive schemes large ratios of $\beta_{0} / \chi_{0}$ really cripple convergence. The reason is that in the strengthened CauchySchwarz inequality of Theorem 4.4 the ratio $\beta_{0} / \chi_{0}$ inevitably occurs.

The classical approach to the analysis of the multigrid method pursued in [4] manages to show that the relative scaling of $\chi_{0}$ and $\beta_{0}$ does not severely affect the rate of convergence, if $\Omega$ and $\Omega_{C}$ coincide and are convex. The techniques applied in this paper cannot deliver on this point, because they cover both multiplicative and additive subspace correction schemes.

\section{CONClusion}

For a setting that is encountered in real eddy current problems, the stability of the nodal multilevel decomposition that gives rise to the hybrid multigrid method for $\boldsymbol{H}(\mathbf{c u r l} ; \Omega)$-elliptic problems has been established. This implies that the rate of convergence of the multigrid method remains bounded away from 1 as $L \rightarrow \infty$. However, the theory does not yet reflect the robustness of the multigrid method with respect to the relative scaling of the parameters $\chi$ and $\beta$. The techniques 
developed in this paper may be powerful devices in the analysis of nonoverlapping domain decomposition methods for edge elements and $\boldsymbol{H}$ (div; $\Omega$ )-elliptic problems.

\section{REFERENCES}

[1] R. Albanese and G. Rubinacci, Analysis of three dimensional electromagnetic fileds using edge elements, J. Comp. Phys., 108 (1993), pp. 236-245.

[2] H. Ammari, A. Buffa, and J.-C. NÉdÉlec, A justification of eddy currents model for the Maxwell equations, SIAM J. Appl. Math., 60 (2000), pp. 1805-1823. MR 2001g:78003

[3] C. Amrouche, C. Bernardi, M. Dauge, and V. Girault, Vector potentials in threedimensional nonsmooth domains, Math. Meth. Appl. Sci., 21 (1998), pp. 823-864. MR 99e:35037

[4] D. Arnold, R. Falk, And R. Winther, Multigrid in $H$ (div) and $H$ (curl), Numer. Math., 85 (2000), pp. 175-195. MR 2001d:65161

[5] E. BÄNsch, Local mesh refinement in 2 and 3 dimensions, IMPACT Comput. Sci. Engrg., 3 (1991), pp. 181-191. MR 92h:65150

[6] R. Beck, P. Deuflhard, R. Hiptmair, R. Hoppe, and B. Wohlmuth, Adaptive multilevel methods for edge element discretizations of Maxwell's equations, Surveys on Mathematics for Industry, 8 (1998), pp. 271-312. MR 2000i:65206

[7] R. BeCK AND R. Hiptmair, Multilevel solution of the time-harmonic Maxwell equations based on edge elements, Int. J. Num. Meth. Engr., 45 (1999), pp. 901-920. MR 2000b:78027

[8] J. BEY, Tetrahedral grid refinement, Computing, 55 (1995), pp. 355-378. MR 96i:65105

[9] M. Birman And M. Solomyak, $L_{2}$-theory of the Maxwell operator in arbitrary domains, Russian Math. Surveys, 42 (1987), pp. 75-96. MR 89e:35127

[10] A. Bonnet-Bendhia, C. Hazard, and S. Lohrengel, A singular field method for the solution of Maxwell's equations in polyhedral domains, SIAM J. Appl. Math., 59 (1999), pp. 2028-2044. MR 2000g:78032

[11] F. Bornemann, A sharpened condition number estimate for the BPX-preconditioner of elliptic finite element problems on highly non-uniform triangulations, Tech. Rep. SC 91-9, ZIB, Berlin, Germany, September 1991.

[12] A. Bossavit, A rationale for edge elements in 3D field computations, IEEE Trans. Mag., 24 (1988), pp. 74-79.

[13] Whitney forms: A class of finite elements for three-dimensional computations in electromagnetism, IEEE Proc. A, 135 (1988), pp. 493-500.

[14] Solving Maxwell's equations in a closed cavity and the question of spurious modes, IEEE Trans. Mag., 26 (1990), pp. 702-705.

[15] _ _ A new viewpoint on mixed elements, Meccanica, 27 (1992), pp. 3-11.

[16] D. Braess and W. HackBusch, A new convergence proof for the multigrid method including the V-cycle, SIAM J. Numer. Anal., 20 (1983), pp. 967-975. MR 85h:65233

[17] J. Bramble, Multigrid methods, vol. 294 of Pitman Research Notes in Mathematics Series, Longman, London, 1993. MR 95b:65002

[18] J. Bramble, J. Pasciak, J. WANG, AND J. Xu, Convergence estimates for multigrid algorithms without regularity assumptions, Math. Comp. 57, 57 (1991), pp. 23-45. MR 91m:65158

[19] J. Bramble and J. Xu, Some estimates for a weighted $L^{2}$-projection, Math. Comp., 56 (1991), pp. 463-476. MR 91k:65140

[20] F. BrezzI, J. Douglas, and D. Marini, Two families of mixed finite elements for 2 nd order elliptic problems, Numer. Math., 47 (1985), pp. 217-235. MR 87g:65133

[21] F. Brezzi And M. Fortin, Mixed and hybrid finite element methods, Springer, 1991. MR 92d:65187

[22] P. Ciarlet, The Finite Element Method for Elliptic Problems, vol. 4 of Studies in Mathematics and its Applications, North-Holland, Amsterdam, 1978. MR 58:25001

[23] P. Ciarlet, Jr. And J. Zou, Fully discrete finite element approaches for time-dependent Maxwell equations, Numer. Math., 82 (1999), pp. 193-219. MR 2000c:65083

[24] M. Clemens and T. Weiland, Transient eddy current calculation with the FI-method, IEEE Trans. Magnetics, 35 (1999), pp. 1163-1166.

[25] G. Cohen And P.Monk, Mur-Nédélec finite element schemes for Maxwell's equations,, Comp. Meth. Appl. Mech. Eng., 169 (1999), p. 197. MR 99k:78002 
[26] M. Costabel and M. Dauge, Singularities of Maxwell's equations on polyhedral domains, in Analysis, Numerics and Applications of Differential and Integral Equations, M. Bach, ed., vol. 379 of Longman Pitman Res. Notes Math. Ser., Addison Wesley, Harlow, 1998, pp. 69-76. CMP 98:09

[27] M. Costabel, M. Dauge, And S. Nicaise, Singularities of Maxwell interface problems, $\mathrm{M}^{2} \mathrm{AN}, 33$ (1999), pp. 627-649. MR 2001g:78005

[28] P. Dular, J.-Y. Hody, A. Nicolet, A. Genon, and W. Legros, Mixed finite elements associated with a collection of tetrahedra, hexahedra and prisms, IEEE Trans Magnetics, MAG-30 (1994), pp. 2980-2983.

[29] V. GiRAult, Curl-conforming finite element methods for Navier-Stokes equations with nonstandard boundary conditions in $\mathbb{R}^{3}$, vol. 1431 of Lecture Notes in Mathematics, SpringerVerlag, Berlin, 1989, pp. 201-218. MR 91k:65143

[30] V. Girault and P. Raviart, Finite element methods for Navier-Stokes equations, Springer, Berlin, 1986. MR 88b:65129

[31] J. Gopalakrishnan AND J. PASCIAK, Overlapping Schwarz preconditioners for indefinite time harmonic Maxwell Equations, Tech. Rep., Department of Mathematics, Texas A\&M University, June 2000. Submitted to Math. Comp.

[32] V. Gradinaru and R. Hiptmair, Whitney elements on pyramids, Electron. Trans. Numer. Anal., 8 (1999), pp. 154-168. MR 2001f:65134

[33] P. Grisvard, Elliptic Problems in Nonsmooth Domains, Pitman, Boston, 1985. MR 86m:35044

[34] R. Hiptmain, Multigrid method for H(div) in three dimensions, ETNA, 6 (1997), pp. 133152. MR 99c:65232

[35] _ Canonical construction of finite elements, Math. Comp., 68 (1999), pp. 1325-1346. MR 2000b:65214

[36] _ Multigrid method for Maxwell's equations, SIAM J. Numer. Anal., 36 (1999), pp. 204-225. MR 99j:65229

[37] R. Hiptmair and R. Hoppe, Multilevel preconditioning for mixed problems in three dimensions, Numer. Math., 82 (1999), pp. 253-279. MR 2000c:65104

[38] R. Hiptmair and K. Neymeyr, Multilevel method for mixed eigenproblems, Report 159, SFB 382, Universität Tübingen, Tübingen, Germany, 2001. To appear in SIAM J. Sci. Comp.

[39] R. Hiptmair, T. Schiekofer, and B. Wohlmuth, Multilevel preconditioned augmented Lagrangian techniques for 2 nd order mixed problems, Computing, 57 (1996), pp. 25-48. MR 98d:65140

[40] R. Hiptmair and A. Toselli, Overlapping and multilevel Schwarz methods for vector valued elliptic problems in three dimensions., in Parallel Solution of Partial Differential Equations, P. Bjorstad and M. Luskin, eds., no. 120 in IMA Volumes in Mathematics and its Applications, Springer, Berlin, 1999, pp. 181-202. MR 2002d:65123

[41] M. HochBruck And C. LUBICH, Error analysis of Krylov methods in a nutshell, SIAM J. Sci. Comput., 19 (1998), pp. 695-701. CMP 98:11

[42] P. KotiugA, On making cuts for magnetic scalar potentials in multiply connected regions, J. Appl. Phys., 61 (1987), pp. 3916-3918.

[43] P. Lions, On the Schwarz alternating method, in Proceedings of the First International Symposium of Domain Decomposition Methods for Partial Differential Equations, R. Glowinski, G. Golub, G. Meurant, and J. Periaux, eds., SIAM, 1987, pp. 1-42. MR 90a:65248

[44] P. Monk, A mixed method for approximating Maxwell's equations, SIAM J. Numer. Anal., 28 (1991), pp. 1610-1634. MR 92j:65173

[45] _ Analysis of a finite element method for Maxwell's equations, SIAM J. Numer. Anal., 29 (1992), pp. 714-729. MR 93k:65096

[46] P. Monk And L. Demkowicz, Discrete compactness and the approximation of Maxwell's equations in $\mathbb{R}^{3}$, Math. Comp., 70 (2001), pp. 507-523. Published online February 23, 2000. MR 2001g:65156

[47] J. NÉDÉLEC, Mixed finite elements in $R^{3}$, Numer. Math., 35 (1980), pp. 315-341. MR 81k:65125

[48] J. NÉDÉLEC, A new family of mixed finite elements in $R^{3}$, Numer. Math., 50 (1986), pp. 5781. MR 88e:65145

[49] P. OswaLd, On function spaces related to the finite element approximation theory, Z. Anal. Anwendungen, 9 (1990), pp. 43-64. MR 91g:65246 
[50] _ Two remarks on multilevel preconditioners, Tech. Rep. 91/1, Methematisches Institut, FSU Jena, 1991.

[51] _ On discrete norm estimates related to multilevel preconditioners in the finite element method, in Constructive Theory of Functions, Proc. Int. Conf. Varna 1991, K. Ivanov, P. Petrushev, and B. Sendov, eds., Bulg. Acad. Sci., 1992, pp. 203-214.

[52] _ On the robustness of the BPX-preconditioner with respect to jumps in the coeffcients, Math. Comp., 68 (1999), pp. 633-650. MR 99i:65143

[53] P. A. Raviart and J. M. Thomas, A Mixed Finite Element Method for Second Order Elliptic Problems, vol. 606 of Springer Lecture Notes in Mathematics, Springer, Ney York, 1977, pp. 292-315. MR 58:3547]

[54] L. R. ScOtT AND Z. ZhANG, Finite element interpolation of nonsmooth functions satisfying boundary conditions, Math. Comp., 54 (1990), pp. 483-493. MR 90j:65021

[55] B. Smith, P. BJørstad, and W. Gropp, Domain decomposition, Cambridge University Press, Cambridge, 1996. MR 98g:65003

[56] A. ToselLI, Overlapping Schwarz methods for Maxwell's equations in three dimensions, Numer. Math., 86 (2000), pp. 733-752. MR 2001h:65137

[57] J. von Neumann, The Geometry of Orhtogonal Spaces Vol II: Functional operators, vol. 22 of Annals of Math. Studies, Princeton University Press, Princeton, 1950. Reprint of lecture notes of 1933. MR 11:599e

[58] H. Whitney, Geometric Integration Theory, Princeton University Press, Princeton, 1957. MR 19:309c

[59] J. Xu, Iterative methods by space decomposition and subspace correction, SIAM Review, 34 (1992), pp. 581-613. MR 93k:65029

[60] _ An introduction to multilevel methods, in Wavelets, Multilevel Methods and Elliptic PDEs, M. Ainsworth, K. Levesley, M. Marletta, and W. Light, eds., Numerical Mathematics and Scientific Computation, Clarendon Press, Oxford, 1997, pp. 213-301. MR 99d:65329

[61] J. XU AND L. ZIKATANOv, The method of alternating projections and the method of subspace corrections in Hilbert space, Report AM 223, Department of Mathematics, PennState University, College Park, PA, June 2000. Submitted.

[62] H. Yserentant, On the multi-level splitting of finite element spaces, Numer. Math., 58 (1986), pp. 379-412. MR 88d:65068a

[63] - Old and new convergence proofs for multigrid methods, Acta Numerica, (1993), pp. 285-326. MR 94i:65128

[64] X. Zhang, Multilevel Schwarz methods, Numer. Math., 63 (1992), pp. 521-539. MR 93h:65047

Sonderforschungsbereich 382, Universität Tübingen, 72076 Tübingen, Germany

E-mail address: hiptmair@na.uni-tuebingen.de

Current address: Seminar für Angewandte Mathematik, ETH Zürich, CH-8092 Zürich, Switzerland

E-mail address: ralf@hiptmair.de 